Dept. of Math./CMA University of Oslo

\title{
A DYNKIN GAME WITH ASYMMETRIC INFORMATION
}

\author{
JUKKA LEMPA AND PEKKA MATOMÄKI
}

\begin{abstract}
We study a Dynkin game with asymmetric information. The game has a random expiry time, which is exponentially distributed and independent of the underlying process. The players have asymmetric information on the expiry time, namely only one of the players is able to observe its occurrence. We propose a set of conditions under which we solve the saddle point equilibrium and study the implications of the information asymmetry. Results are illustrated with an explicit example.
\end{abstract}

\section{INTRODUCTION}

Dynkin games are game variants of optimal stopping problems, for the seminal study see [6]. Such a game has two players, "buyer" and "issuer", and both of them can stop the underlying process prior the terminal time. In this paper we study the following formulation of the game. First, we assume that the underlying process $X$ is a time homogenous diffusion; we will elaborate the assumptions on $X$ in the next section. At the initial time $t=0$, the players choose their own stopping times $\tau$ (buyer) and $\gamma$ (issuer) and at the time of the first exercise, i.e. at $\tau \wedge \gamma$, the issuer pays the buyer the amount

$$
g_{1}\left(X_{\tau}\right) \mathbf{1}_{\{\tau<\gamma\}}+g_{2}\left(X_{\gamma}\right) \mathbf{1}_{\{\tau>\gamma\}}+g_{3}\left(X_{\gamma}\right) \mathbf{1}_{\{\tau=\gamma\}}
$$

we will pose assumptions on the payoff functions $g_{i}$ in the next section. An interpretation of this is that, at any stopping time $\gamma$, the issuer can cancel the buyer's right to exercise, but she has to pay the cost $g_{2}\left(X_{\gamma}\right)$ to do so. Now, it is the buyers (issuers) objective to choose the stopping time $\tau(\gamma)$ such that the expected

2010 Mathematics Subject Classification. 60G40,60J60.

Key words and phrases. Dynkin game, Nash equilibrium, linear diffusion, resolvent operator, Poisson process.

Address. Jukka Lempa, Centre of Mathematics for Applications, University of Oslo, PO Box 1053 Blindern, NO - 0316 Oslo, e-mail: jlempa@cma.uio.no

Pekka Matomäki, Department of Accounting and Finance, Turku School of Economics, University of Turku, Rehtorinpellonkatu 3, FIN - 20500 Turku, e-mail: pjsila@utu.fi. 
present value of the exercise payoff

$$
\Pi(x, \tau, \gamma)=\mathbf{E}_{x}\left\{e^{-r(\tau \wedge \gamma)}\left[g_{1}\left(X_{\tau}\right) \mathbf{1}_{\{\tau<\gamma\}}+g_{2}\left(X_{\gamma}\right) \mathbf{1}_{\{\tau>\gamma\}}+g_{3}\left(X_{\gamma}\right) \mathbf{1}_{\{\tau=\gamma\}}\right]\right\}
$$

is maximized (minimized). Here, $r>0$ is the constant rate of discounting.

The objective of this paper is to study a version of this game with random time horizon, the infinite horizon game given by the expression (1.2) being already analysed comprehensively e.g. in [1] and [8]. In financial terminology the random time horizon game can be interpreted as a perpetual game option with default risk, for studies on game options see e.g. [7], [12] and [13]. We remark that our problem can be regarded also as a Canadized version of a finite horizon game - for studies considering Canadization of options, see [4], [13] and [14]. To introduce the random time horizon, we assume that, in addition to the diffusion $X$, there is also an independent Poisson process $N$ defined on the underlying probability space. Furthermore, we assume that the game expires at the first jump time of the Poisson process, that is we assume that the game has an exponentially distributed random time horizon. The existence of the terminating event and its rate is assumed to be known to the players, while the information of it is asymmetric: we assume that the occurrence of the expiring event is observable only to one of the players. Here, the information asymmetry has an interpretation as inside information. Indeed, the player who observes the default taking place has more information than is commonly available on the market and can be considered as an insider. We make a distinction between the cases when either buyer (Game 1) or issuer (Game 2) observes the jump of the Poisson process and study both of these cases separately.

Our approach to the problem is built on Markovian approach to Dynkin games. There is a substantial literature in this area highlighting various parts of the theory. For instance, studies [1] and [2] are concerned with deriving explicit characterization for the value and saddle point equilibrium using classical theory of diffusions and standard nonlinear programming techniques. A generalized concavity approach is used in [7] and [8] to produce the optimal solution via the theory of excessive functions. In [9] and [17], the authors study equilibrium properties of Dynkin games under very general Markovian setup. Our setup and approach is closely related to [1] and can be regarded as a partial extension of it. We start our analysis by first deriving partly heuristically a free boundary problem which gives us a candidate for the solution. To set up the free boundary problem, we assume that the optimal continuation region is an interval with compact closure with constant thresholds. Given the time homogeneity of the diffusion $X$ and the fact that the discount rate $r$ and the jump rate of $N$ are constants, this is indeed a reasonable assumption. 
We derive necessary and sufficient conditions for the existence of a unique Nash equilibrium for Games 1 and 2. We also carry out a comparison of the solutions showing that whenever Games 1 and 2 have a saddle point solution, the value of Game 1 dominates the value of Game 2. Furthermore, we show that if the payoff $g_{2}$ is non-negative, the value of the infinite horizon game dominates both the value of Game 1 and 2 . Interestingly, we find that if $g_{2}$ admits also negative values, then the value of the infinite horizon game can even be the smallest of the three. We discuss also the symmetric information case where the expiring event is not observable to either of the players - denote this as Game 3. In this case, we find that the value is in between the values of Game 1 and Game 2. We also show that the optimal continuation regions of Games 1 - 3 are related in a way that can be described as follows: If you are able to observe the terminating event, you will wait longer - The more you know, the longer you wait.

The reminder of the paper is organized as follows. In Section 2 we set up the underlying dynamics and introduce the Dynkin games. In Sections 3 and 4 we study the solvability of the games and discuss some implications of the information asymmetry. In Section 5 we compare the optimal solutions of the games and study limiting behavior of the solutions. In Section 6 we illustrate the main results of the study with an explicit example.

\section{The Games}

2.1. Underlying Dynamics. Let $(\Omega, \mathcal{F}, \mathbb{F}, \mathbf{P})$, with $\mathbb{F}=\left\{\mathcal{F}_{t}\right\}_{t \geq 0}$, be a complete filtered probability space satisfying the usual conditions, see [3], p. 2. In addition, let $W$ be a Wiener process on $(\Omega, \mathcal{F}, \mathbb{F}, \mathbf{P})$. We assume that the state process $X$ is a regular linear diffusion defined on $(\Omega, \mathcal{F}, \mathbb{F}, \mathbf{P})$, evolving on $\mathbf{R}_{+}$, and given as the solution of the Itô equation

$$
d X_{t}=\mu\left(X_{t}\right) d t+\sigma\left(X_{t}\right) d W_{t}, X_{0}=x
$$

where the coefficients $\mu: \mathbf{R}_{+} \rightarrow \mathbf{R}$ and $\sigma: \mathbf{R}_{+} \rightarrow \mathbf{R}_{+}$are assumed to be sufficiently smooth to guarantee the existence of a unique (weak) solution of (2.1), see [3], pp. 46-47. In line with most economical and financial applications, we assume that $X$ does not die inside the state space $\mathbf{R}_{+}$, i.e., that killing of $X$ is possible only at the boundaries 0 and $\infty$. Therefore the boundaries 0 and $\infty$ are either natural, entrance, exit or regular. In the case a boundary is regular, it is assumed to be killing, see [3], pp. 18-20, for a characterization of the boundary behavior of diffusions. The assumption that the state space is $\mathbf{R}_{+}$is done for reasons of notational convenience. In fact, we could assume that the state space is any interval $\mathcal{I}$ in $\mathbf{R}$ and all our subsequent analysis would hold with obvious modifications. Denote as $\mathcal{A}=\frac{1}{2} \sigma^{2}(x) \frac{d^{2}}{d x^{2}}+\mu(x) \frac{d}{d x}$ the differential operator associated to the process $X$. For notational convenience we denote $\mathcal{G}_{\beta}=\mathcal{A}-\beta$ for a given constant $\beta>0$. 
For any given constant $\beta>0$, we denote as $\mathcal{L}_{1}^{\beta}$ the class of real valued measurable functions $f$ on $\mathbf{R}_{+}$ satisfying the condition

$$
\mathbf{E}_{x}\left\{\int_{0}^{\zeta} e^{-\beta t}\left|f\left(X_{t}\right)\right| d t\right\}<\infty,
$$

where $\zeta:=\inf \left\{t>0: X_{t} \notin \mathbf{R}_{+}\right\}$denotes the lifetime of $X$. In addition, for any given constant $\beta>0$, we denote, respectively, as $\psi_{\beta}$ and $\varphi_{\beta}$ the increasing and the decreasing solution of the ordinary second-order linear differential equation $\mathcal{G}_{\beta} u(x)=0$ defined on the domain of the characteristic operator of $X$ - for the characterization and fundamental properties of the minimal $\beta$-excessive functions $\psi_{\beta}$ and $\varphi_{\beta}$, see [3], pp. 18-20. Denote as $B_{\beta}=\frac{\psi_{\beta}^{\prime}(x)}{S^{\prime}(x)} \varphi_{\beta}(x)-\frac{\varphi_{\beta}^{\prime}(x)}{S^{\prime}(x)} \psi_{\beta}(x)$ the Wronskian determinant, where

$$
S^{\prime}(x)=\exp \left(-\int^{x} \frac{2 \mu(y)}{\sigma^{2}(y)} d y\right)
$$

denotes the density of the scale function of $X$, see [3], p. 19. We remark that the value of the Wronskian does not depend on the initial state $x$ but on the constant $\beta$. For a function $f \in \mathcal{L}_{1}^{\beta}$, the resolvent $R_{\beta} f: \mathbf{R}_{+} \rightarrow \mathbf{R}$ is defined as

$$
\left(R_{\beta} f\right)(x)=\mathbf{E}_{x}\left\{\int_{0}^{\zeta} e^{-\beta t} f\left(X_{t}\right) d t\right\},
$$

for all $x \in \mathbf{R}_{+}$. The resolvent $R_{\beta}$ and the solutions $\psi_{\beta}$ and $\varphi_{\beta}$ are connected in a computationally very useful way. Indeed, we know from the literature, see [3], pp. $17-20$ and p. 29, that for given $f \in \mathcal{L}_{1}^{\beta}$ the resolvent $R_{\beta} f$ can be expressed as

$$
\left(R_{\beta} f\right)(x)=B_{\beta}^{-1} \varphi_{\beta}(x) \int_{0}^{x} \psi_{\beta}(y) f(y) m^{\prime}(y) d y+B_{\beta}^{-1} \psi_{\beta}(x) \int_{x}^{\infty} \varphi_{\beta}(y) f(y) m^{\prime}(y) d y,
$$

for all $x \in \mathbf{R}_{+}$, where $m^{\prime}(x)=\frac{2}{\sigma^{2}(x) S^{\prime}(x)}$ denotes the speed density of $X$.

To close the subsection, we denote as $N$ a Poisson process with intensity $\lambda>0$, and assume that $N$ is independent of the underlying $X$. Now, the first jump time $T$ of $N$ is an exponentially distributed random time with mean $\frac{1}{\lambda}$. Denote as $\hat{\mathbb{F}}=\left\{\hat{\mathcal{F}}_{t}\right\}_{t \geq 0}$ the enlarged filtration defined as $\hat{\mathcal{F}}_{t}=\mathcal{F}_{t} \vee \sigma(\{T \leq s\}: s \leq t)$. In other words, the filtration $\hat{\mathbb{F}}$ carries the information of the evolution of underlying $X$ and the first jump of the Poisson process $N$. We denote as $\mathcal{T}_{0}$ as the set of all $\mathbb{F}$-stopping times and as $\mathcal{T}_{1}$ the set $\mathcal{T}_{0}$ augmented with $T$, i.e., the set of all $\hat{\mathbb{F}}$-stopping times.

2.2. The Games. Dynkin game is an optimal stopping game between two players, "buyer" and "issuer". In contrast to classical optimal stopping problems, also the issuer can now exercise. Recall now the definition 
of the expected present value of the exercise payoff from (1.2). Throughout the study, we make the following standing assumptions for the payoffs $g_{i}$.

Assumption 2.1. We assume that the payoffs $g_{i}: \mathbf{R}_{+} \rightarrow \mathbf{R}, i=1,2,3$, are continuous and non-decreasing functions satisfying the ordering $g_{1} \leq g_{3} \leq g_{2}$ and that $g_{1}$ is bounded from below. Furthermore, we assume that $g_{i} \in \mathcal{L}_{1}^{r}$ and $g_{i} \in C^{1}\left(\mathbf{R}_{+}\right) \cap C^{2}\left(\mathbf{R}_{+} \backslash D\right)$, where the set $D$ is finite.

In order to propose a value and notions of equilibrium for the considered games, define first the lower and upper values $\underline{V}$ and $\bar{V}$ as

$$
\underline{V}(x)=\sup _{\tau \in \mathcal{T}} \inf _{\gamma \in \mathcal{T}} \Pi(x, \tau, \gamma), \bar{V}(x)=\inf _{\gamma \in \mathcal{T}} \sup _{\tau \in \mathcal{T}} \Pi(x, \tau, \gamma)
$$

where $\mathcal{T}$ is the class of admissible stopping times. Following [8], pp. 1578, we remark that $g_{1} \leq \underline{V} \leq \bar{V} \leq g_{2}$. If, on the other hand, the values satisfy $\underline{V} \geq \bar{V}$, we say that the game has the value $V:=\underline{V}=\bar{V}$, i.e. has a Stackelberg equilibrium. Moreover, if there exists stopping times $\tau^{*}$ and $\gamma^{*}$ such that

$$
\Pi\left(x, \tau, \gamma^{*}\right) \leq \Pi\left(x, \tau^{*}, \gamma^{*}\right) \leq \Pi\left(x, \tau^{*}, \gamma\right)
$$

for all $x \in \mathbf{R}_{+}$, then the pair $\left(\tau^{*}, \gamma^{*}\right)$ constitutes the saddle point, i.e., the Nash equilibrium of the game. We remark that the existence of the saddle point implies the existence of the value but the converse does not hold in general - for a study addressing this problem in a general Markovian setting, see [9]. However, in our setting the underlying process is nice enough so that Stackelberg equilibrium is equivalent to Nash equilibrium.

The main objective of this paper is to study two Dynkin games which are associated via a certain type of information asymmetry. To make a precise statement, recall the Poisson process $N$ from the previous section. At the initial time $t=0$, the underlying $X$ and exogenous $N$ are both started. At the first jump time $T$, the game ends. Thus, the considered games have an exponentially distributed random time horizon which is independent of $X$. The information asymmetry is introduced as follows: we assume that the occurrence of the expiring event is observable only to one of the players. Let us formalize this setting first in the case when $T$ is observable to the buyer; later this case will be referred to as Game 1. First, recall the definitions of the sets $\mathcal{T}_{0}$ and $\mathcal{T}_{1}$ from the previous subsection. At the start of the game, issuer choose a stopping time from set $\mathcal{T}_{0}$ and the buyer from the set $\mathcal{T}_{1}$. The expected present value $\Pi_{1}$ of the exercise payoff is written as

$$
\Pi_{1}(x, \tau, \gamma)=\mathbf{E}_{x}\left\{e^{-r(\tau \wedge \gamma)}\left[g_{1}\left(X_{\tau}\right) \mathbf{1}_{\{\tau<\gamma\}}+g_{2}\left(X_{\gamma}\right) \mathbf{1}_{\{\tau>\gamma\}}+g_{3}\left(X_{\gamma}\right) \mathbf{1}_{\{\tau=\gamma\}}\right] \mathbf{1}_{\{\tau \wedge \gamma \leq T\}}\right\},
$$


and the upper and lower values are defined as

$$
\underline{V}_{1}(x)=\sup _{\tau \in \mathcal{T}_{1}} \inf _{\gamma \in \mathcal{T}_{0}} \Pi_{1}(x, \tau, \gamma), \bar{V}_{1}(x)=\inf _{\gamma \in \mathcal{T}_{0}} \sup _{\tau \in \mathcal{T}_{1}} \Pi_{1}(x, \tau, \gamma)
$$

For Game 1, we denote the value function as $V_{1}$ and the saddle point equilibrium as $\left(\tau_{1}^{*}, \gamma_{1}^{*}\right)$.

The setup of the second game, which will be referred to as Game 2, is completely analogous. For Game 2, we assume that the random time $T$ is a stopping time to issuer. Similarly to Game 1, we define the expected present value $\Pi_{2}$ of the exercise payoff as

$$
\Pi_{2}(x, \tau, \gamma)=\mathbf{E}_{x}\left\{e^{-r(\tau \wedge \gamma)}\left[g_{1}\left(X_{\tau}\right) \mathbf{1}_{\{\tau<\gamma\}}+g_{2}\left(X_{\gamma}\right) \mathbf{1}_{\{\tau>\gamma\}}+g_{3}\left(X_{\gamma}\right) \mathbf{1}_{\{\tau=\gamma\}}\right] \mathbf{1}_{\{\gamma \wedge \tau \leq T\}}\right\}
$$

and the upper and lower values are defined as

$$
\underline{V}_{2}(x)=\sup _{\tau \in \mathcal{T}_{0}} \inf _{\gamma \in \mathcal{T}_{1}} \Pi_{2}(x, \tau, \gamma), \bar{V}_{2}(x)=\inf _{\gamma \in \mathcal{T}_{1}} \sup _{\tau \in \mathcal{T}_{0}} \Pi_{2}(x, \tau, \gamma)
$$

Analogously to Game 1, the value function of Game 2 is denoted as $V_{2}$ and the saddle point equilibrium as $\left(\tau_{2}^{*}, \gamma_{2}^{*}\right)$.

\section{Game 1}

3.1. Equivalent formulation of the game. First, we introduce some additional definitions and notations. Following [1] (see also [19]), define the operators $L_{\psi}^{\beta}$ and $L_{\varphi}^{\beta}$ for sufficiently smooth functions $f: \mathbf{R}_{+} \rightarrow \mathbf{R}$ as

$$
\left\{\begin{array}{l}
\left(L_{\psi}^{\beta} f\right)(x)=\frac{f^{\prime}(x)}{S^{\prime}(x)} \psi_{\beta}(x)-\frac{\psi_{\beta}^{\prime}(x)}{S^{\prime}(x)} f(x), \\
\left(L_{\varphi}^{\beta} f\right)(x)=\frac{f^{\prime}(x)}{S^{\prime}(x)} \varphi_{\beta}(x)-\frac{\varphi_{\beta}^{\prime}(x)}{S^{\prime}(x)} f(x),
\end{array}\right.
$$

for a given constant $\beta>0$. In order to simplify the upcoming notation, define the functions $\hat{g}_{i}: \mathbf{R}_{+} \rightarrow \mathbf{R}$, $i=1,2$, as

$$
\left\{\begin{array}{l}
\hat{g}_{1}(x)=g_{1}(x)-\lambda\left(R_{r+\lambda} g_{1}^{+}\right)(x), \\
\hat{g}_{2}(x)=g_{2}(x)-\lambda\left(R_{r+\lambda} g_{1}^{+}\right)(x),
\end{array}\right.
$$

where $g_{1}^{+}(x)=\max \left\{g_{1}(x), 0\right\}$. We remark that since we assumed $g_{1} \leq g_{2}$, also $\hat{g}_{1} \leq \hat{g}_{2}$.

In this subsection, we transform Game 1 into an adjusted perpetual game and study its solvability. To this end, we derive first a candidate $G_{1}$ for the optimal value function in a partly heuristic way - for a related study in a different context, see [10]. We start with the ansatz that the game has a saddle point equilibrium. Because the exponential distribution has memoryless property and the underlying dynamic structure is time 
homogeneous, we assume that the state space $\mathbf{R}_{+}$is partitioned into continuation and action regions, where the continuation region $\left(z_{1}^{*}, y_{1}^{*}\right) \subset \mathbf{R}_{+}$has compact closure. If $x \in\left(z_{1}^{*}, y_{1}^{*}\right)$, the players wait by definition. Now, in an infinitesimal time interval $d t$, the Poisson process jumps (expiring the exercise opportunities) with probability $\lambda d t$. Because the buyer can exercise at time $T$, she will exercise at that time if and only if $g_{1} \geq 0$; this yields the terminal payoff $g_{1}^{+}(x)$. On the other hand, with probability $1-\lambda d t$ the contract lives on yielding additional expected present value. Denote as $G_{1}$ the candidate for the value function. Formally, this suggests with a heuristic use of Dynkin's theorem, see e.g. [16], that

$$
\begin{aligned}
G_{1}(x) & =g_{1}^{+}(x) \lambda d t+(1-\lambda d t) \mathbf{E}_{x}\left[e^{-r d t} G_{1}\left(X_{d t}\right)\right]=\lambda g_{1}^{+}(x) d t+(1-\lambda d t)\left[G_{1}(x)+\mathcal{G}_{r} G_{1}(x) d t\right] \\
& =G_{1}(x)+\mathcal{G}_{r} G_{1}(x) d t+\lambda\left(g_{1}^{+}(x)-G_{1}(x)\right)
\end{aligned}
$$

for all $x \in\left(z_{1}^{*}, y_{1}^{*}\right)$ under the intuition $d t^{2}=0$. This yields the condition

$$
\mathcal{G}_{r+\lambda} G_{1}(x)=-\lambda g_{1}^{+}(x)
$$

for all $x \in\left(z_{1}^{*}, y_{1}^{*}\right)$. The solutions of the equation (3.3) can be expressed as $G_{1}(x)=\lambda\left(R_{r+\lambda} g_{1}^{+}\right)(x)+$ $c_{1} \psi_{r+\lambda}(x)+c_{2} \varphi_{r+\lambda}(x)$ for some positive constants $c_{1}$ and $c_{2}$. We assume that the candidate $G_{1}$ satisfies the value-matching condition, i.e., is continuous over the boundary of $\left(z_{1}^{*}, y_{1}^{*}\right)$. This condition can be expressed as

$$
\left\{\begin{array}{l}
\lambda\left(R_{r+\lambda} g_{1}^{+}\right)\left(z_{1}^{*}\right)+c_{1} \psi_{r+\lambda}\left(z_{1}^{*}\right)+c_{2} \varphi_{r+\lambda}\left(z_{1}^{*}\right)=g_{2}\left(z_{1}^{*}\right), \\
\lambda\left(R_{r+\lambda} g_{1}^{+}\right)\left(y_{1}^{*}\right)+c_{1} \psi_{r+\lambda}\left(y_{1}^{*}\right)+c_{2} \varphi_{r+\lambda}\left(y_{1}^{*}\right)=g_{1}\left(y_{1}^{*}\right) .
\end{array}\right.
$$

Using the notation from (3.2), it is a matter of elementary algebra to show that

$$
\left\{\begin{array}{l}
c_{1}=\frac{\varphi_{r+\lambda}\left(y_{1}^{*}\right) \hat{g}_{2}\left(z_{1}^{*}\right)-\varphi_{r+\lambda}\left(z_{1}^{*}\right) \hat{g}_{1}\left(y_{1}^{*}\right)}{\varphi_{r+\lambda}\left(y_{1}^{*}\right) \psi_{r+\lambda}\left(z_{1}^{*}\right)-\varphi_{r+\lambda}\left(z_{1}^{*}\right) \psi_{r+\lambda}\left(y_{1}^{*}\right)}:=h_{1}\left(z_{1}^{*}, y_{1}^{*}\right) \\
c_{2}=\frac{\psi_{r+\lambda}\left(z_{1}^{*}\right) \hat{g}_{1}\left(y_{1}^{*}\right)-\psi_{r+\lambda}\left(y_{1}^{*}\right) \hat{g}_{2}\left(z_{1}^{*}\right)}{\varphi_{r+\lambda}\left(y_{1}^{*}\right) \psi_{r+\lambda}\left(z_{1}^{*}\right)-\varphi_{r+\lambda}\left(z_{1}^{*}\right) \psi_{r+\lambda}\left(y_{1}^{*}\right)}:=h_{2}\left(z_{1}^{*}, y_{1}^{*}\right) .
\end{array}\right.
$$

To proceed, denote as $\tau_{\left(z_{1}^{*}, y_{1}^{*}\right)}$ the first exit time of $X$ from the interval $\left(z_{1}^{*}, y_{1}^{*}\right)$. We know from [5], Theorem 13.11, p. 46-47, that the function $x \mapsto \mathbf{E}_{x}\left[e^{-(r+\lambda) \tau_{\left(z_{1}^{*}, y_{1}^{*}\right)}}\right]$ solves the boundary value problem $\mathcal{G}_{r+\lambda} u(x)=0$ on $\left(z_{1}^{*}, y_{1}^{*}\right)$ with boundary conditions $u\left(z_{1}^{*}\right)=u\left(y_{1}^{*}\right)=1$. Using this, we find that

$$
\begin{aligned}
& \mathbf{E}_{x}\left\{e^{-(r+\lambda)\left(\tau_{y_{1}^{*}} \wedge \gamma_{z_{1}^{*}}\right)} \mathbf{1}_{\left\{\tau_{y_{1}^{*}}<\gamma_{z_{1}^{*}}\right\}}\right\}=\frac{\varphi_{r+\lambda}(x) \psi_{r+\lambda}\left(z_{1}^{*}\right)-\varphi_{r+\lambda}\left(z_{1}^{*}\right) \psi_{r+\lambda}(x)}{\varphi_{r+\lambda}\left(y_{1}^{*}\right) \psi_{r+\lambda}\left(z_{1}^{*}\right)-\varphi_{r+\lambda}\left(z_{1}^{*}\right) \psi_{r+\lambda}\left(y_{1}^{*}\right)}, \\
& \mathbf{E}_{x}\left\{e^{-(r+\lambda)\left(\tau_{\left.y_{1}^{*} \wedge \gamma_{1}^{*}\right)}\right.} \mathbf{1}_{\left\{\tau_{y_{1}^{*}}>\gamma_{z_{1}^{*}}\right\}}\right\}=\frac{\varphi_{r+\lambda}\left(y_{1}^{*}\right) \psi_{r+\lambda}(x)-\varphi_{r+\lambda}(x) \psi_{r+\lambda}\left(y_{1}^{*}\right)}{\varphi_{r+\lambda}\left(y_{1}^{*}\right) \psi_{r+\lambda}\left(z_{1}^{*}\right)-\varphi_{r+\lambda}\left(z_{1}^{*}\right) \psi_{r+\lambda}\left(y_{1}^{*}\right)},
\end{aligned}
$$


see also [15]. Consequently, the candidate $G_{1}$ can be rewritten as

$$
\begin{aligned}
G_{1}(x)=\lambda\left(R_{r+\lambda} g_{1}^{+}\right)(x) & +\hat{g}_{1}\left(y_{1}^{*}\right) \mathbf{E}_{x}\left\{e^{-(r+\lambda)\left(\tau_{y_{1}^{*}} \wedge \gamma_{z_{1}^{*}}\right)} \mathbf{1}_{\left\{\tau_{y_{1}^{*}}<\gamma_{z_{1}^{*}}\right\}}\right\} \\
& +\hat{g}_{2}\left(z_{1}^{*}\right) \mathbf{E}_{x}\left\{e^{-(r+\lambda)\left(\tau_{y_{1}^{*}} \wedge \gamma_{z_{1}^{*}}\right)} \mathbf{1}_{\left\{\tau_{y_{1}^{*}}>\gamma_{z_{1}^{*}}\right\}}\right\}
\end{aligned}
$$

for all $x \in\left(z_{1}^{*}, y_{1}^{*}\right)$. Since the sample paths of $X$ are (almost surely) continuous, an application of the strong Markov property of the underlying $X$ yields

$$
G_{1}(x)=\mathbf{E}_{x}\left\{\lambda \int_{0}^{\tau_{y_{1}^{*}} \wedge \gamma_{z_{1}^{*}}} e^{-(r+\lambda) s} g_{1}^{+}\left(X_{s}\right) d s+e^{-(r+\lambda)\left(\tau_{y_{1}^{*}} \wedge \gamma_{z_{1}^{*}}\right)}\left[g_{1}\left(X_{\left.\tau_{y_{1}^{*}}\right)} \mathbf{1}_{\left\{\tau_{y_{1}^{*}}<\gamma_{z_{1}^{*}}\right\}}+g_{2}\left(X_{{\gamma_{1}^{*}}_{1}^{*}}\right) \mathbf{1}_{\left\{\tau_{y_{1}^{*}}>\gamma_{z_{1}^{*}}\right\}}\right]\right\}\right.
$$

for all $x \in \mathbf{R}_{+}$. This result indicates the form of the equivalent perpetual game. The next proposition confirms that this partly heuristic derivation gives the correct form of the adjusted perpetual problem. For a rigorous proof we though need an auxiliary lemma.

Lemma 3.1. For $\tau \in \mathcal{T}_{1}$, there exists $\tau^{\prime} \in \mathcal{T}_{0}$ such that $\tau \wedge T=\tau^{\prime} \wedge T$ a.s.

Proof. See [18], Lemma, Section VI.3, p. 378.

Proposition 3.2. The upper and lower values for Game 1 can be rewritten as

$$
\bar{V}_{1}(x)=\inf _{\gamma \in \mathcal{T}_{0}} \sup _{\tau \in \mathcal{T}_{0}} \tilde{\Pi}_{1}(x, \tau, \gamma), \underline{V}_{1}(x)=\sup _{\tau \in \mathcal{T}_{0}} \inf _{\gamma \in \mathcal{T}_{0}} \tilde{\Pi}_{1}(x, \tau, \gamma)
$$

where

$\tilde{\Pi}_{1}(x, \tau, \gamma)=\mathbf{E}_{x}\left\{\lambda \int_{0}^{\tau \wedge \gamma} e^{-(r+\lambda) s} g_{1}^{+}\left(X_{s}\right) d s+e^{-(r+\lambda)(\tau \wedge \gamma)}\left[g_{1}\left(X_{\tau}\right) \mathbf{1}_{\{\tau<\gamma\}}+g_{2}\left(X_{\gamma}\right) \mathbf{1}_{\{\tau>\gamma\}}+g_{3}\left(X_{\gamma}\right) \mathbf{1}_{\{\tau=\gamma\}}\right]\right\}$

for all $x \in \mathbf{R}_{+}$.

Proof. Let $\hat{\mathcal{T}}_{1}$ denote the set containing $\hat{\mathbb{F}}$-stopping times satisfying $\tau \leq T$ for all $\omega$. We know that for all $\tau \in \hat{\mathcal{T}}_{1}$, there is a $\tau^{\prime} \in \mathcal{T}_{1}$ for which $\tau^{\prime}=\tau \wedge T$. Because buyer's objective is to maximize the expected present value of the payoff and she is aware that after the observable expiry time $T$ the payoff will be zero, we reason 
that

$$
\begin{aligned}
V_{1}(x) & =\sup _{\tau \in \mathcal{T}_{1}} \inf _{\gamma \in \mathcal{T}_{0}} \mathbf{E}_{x}\left\{e^{-r(\tau \wedge \gamma)}\left[g_{1}\left(X_{\tau}\right) \mathbf{1}_{\{\tau<\gamma\}}+g_{2}\left(X_{\gamma}\right) \mathbf{1}_{\{\tau>\gamma\}}+g_{3}\left(X_{\gamma}\right) \mathbf{1}_{\{\tau=\gamma\}}\right] \mathbf{1}_{\{\tau \wedge \gamma \leq T\}}\right\} \\
& =\sup _{\tau \in \mathcal{T}_{1}} \inf _{\gamma \in \mathcal{T}_{0}} \mathbf{E}_{x}\left\{e^{-r(\tau \wedge \gamma)}\left[\left(g_{1}\left(X_{\tau}\right) \mathbf{1}_{\{\tau<T\}}+g_{1}^{+}\left(X_{T}\right) \mathbf{1}_{\{\tau \geq T\}}\right) \mathbf{1}_{\{\tau<\gamma\}}+g_{2}\left(X_{\gamma}\right) \mathbf{1}_{\{\tau>\gamma\}}+g_{3}\left(X_{\gamma}\right) \mathbf{1}_{\{\tau=\gamma\}}\right] \mathbf{1}_{\{\tau \wedge \gamma \leq T\}}\right\} \\
& =\sup _{\hat{\tau} \in \hat{\mathcal{T}}_{1}} \inf _{\gamma \in \mathcal{T}_{0}} \mathbf{E}_{x}\left\{e^{-r(\hat{\tau} \wedge \gamma)}\left[\left(g_{1}\left(X_{\hat{\tau}}\right) \mathbf{1}_{\{\hat{\tau}<T\}}+g_{1}^{+}\left(X_{T}\right) \mathbf{1}_{\{\hat{\tau} \geq T\}}\right) \mathbf{1}_{\{\hat{\tau}<\gamma\}}+g_{2}\left(X_{\gamma}\right) \mathbf{1}_{\{\hat{\tau}>\gamma\}}+g_{3}\left(X_{\gamma}\right) \mathbf{1}_{\{\hat{\tau}=\gamma\}}\right] \mathbf{1}_{\{\hat{\tau} \wedge \gamma \leq T\}}\right\} \\
& =\sup _{\hat{\tau} \in \hat{\mathcal{T}}_{1}} \inf _{\gamma \in \mathcal{T}_{0}} \mathbf{E}_{x}\left\{e^{-r(\hat{\tau} \wedge \gamma)}\left[\left(g_{1}\left(X_{\hat{\tau}}\right) \mathbf{1}_{\{\hat{\tau}<T\}}+g_{1}^{+}\left(X_{T}\right) \mathbf{1}_{\{\hat{\tau} \geq T\}}\right) \mathbf{1}_{\{\hat{\tau}<\gamma\}}+g_{2}\left(X_{\gamma}\right) \mathbf{1}_{\{\hat{\tau}>\gamma\}}+g_{3}\left(X_{\gamma}\right) \mathbf{1}_{\{\hat{\tau}=\gamma\}}\right]\right\} \\
& =\sup _{\tau \in \mathcal{T}_{1}} \inf _{\gamma \in \mathcal{T}_{0}} \mathbf{E}_{x}\left\{e^{-r((\tau \wedge T) \wedge \gamma)}\left[\left(g_{1}\left(X_{\tau}\right) \mathbf{1}_{\{\tau<T\}}+g_{1}^{+}\left(X_{T}\right) \mathbf{1}_{\{\tau \geq T\}}\right) \mathbf{1}_{\{\tau \wedge T<\gamma\}}+g_{2}\left(X_{\gamma}\right) \mathbf{1}_{\{\tau \wedge T>\gamma\}}+g_{3}\left(X_{\gamma}\right) \mathbf{1}_{\{\tau \wedge T=\gamma\}}\right]\right\}
\end{aligned}
$$

Now, it follows from Lemma 3.1 that the last expression is equivalent with the form

$\left.\sup _{\tau \in \mathcal{T}_{0}} \inf _{\gamma \in \mathcal{T}_{0}} \mathbf{E}_{x}\left\{e^{-r((\tau \wedge T) \wedge \gamma)}\left[\left(g_{1}\left(X_{\tau}\right) \mathbf{1}_{\{\tau<T\}}+g_{1}^{+}\left(X_{T}\right) \mathbf{1}_{\{\tau \geq T\}}\right)\right) \mathbf{1}_{\{\tau \wedge T<\gamma\}}+g_{2}\left(X_{\gamma}\right) \mathbf{1}_{\{\tau \wedge T>\gamma\}}+g_{3}\left(X_{\gamma}\right) \mathbf{1}_{\{\tau \wedge T=\gamma\}}\right]\right\}$.

Finally, let $\tau, \gamma \in \mathcal{T}_{0}$. Since $T$ is independent of $X$, we conclude that

$$
\begin{aligned}
& \left.\mathbf{E}_{x}\left\{e^{-r((\tau \wedge T) \wedge \gamma)}\left[\left(g_{1}\left(X_{\tau}\right) \mathbf{1}_{\{\tau<T\}}+g_{1}^{+}\left(X_{T}\right) \mathbf{1}_{\{\tau \geq T\}}\right)\right) \mathbf{1}_{\{\tau \wedge T<\gamma\}}+g_{2}\left(X_{\gamma}\right) \mathbf{1}_{\{\tau \wedge T>\gamma\}}+g_{3}\left(X_{\gamma}\right) \mathbf{1}_{\{\tau \wedge T=\gamma\}}\right]\left(\mathbf{1}_{\{\tau \geq T\}}+\mathbf{1}_{\{\tau<T\}}\right)\right\} \\
= & \mathbf{E}_{x}\left\{e^{-r T} g_{1}^{+}\left(X_{T}\right) \mathbf{1}_{\{\tau \wedge \gamma \geq T\}}+e^{-r(\tau \wedge \gamma)}\left[g_{1}\left(X_{\tau}\right) \mathbf{1}_{\{\tau<\gamma\}}+g_{2}\left(X_{\gamma}\right) \mathbf{1}_{\{\tau>\gamma\}}+g_{3}\left(X_{\gamma}\right) \mathbf{1}_{\{\tau=\gamma\}}\right] \mathbf{1}_{\{\tau \wedge \gamma<T\}}\right\} \\
= & \mathbf{E}_{x}\left\{\lambda \int_{0}^{\tau \wedge \gamma} e^{-(r+\lambda) s} g_{1}^{+}\left(X_{s}\right) d s+e^{-(r+\lambda)(\tau \wedge \gamma)}\left[g_{1}\left(X_{\tau}\right) \mathbf{1}_{\{\tau<\gamma\}}+g_{2}\left(X_{\gamma}\right) \mathbf{1}_{\{\tau>\gamma\}}+g_{3}\left(X_{\gamma}\right) \mathbf{1}_{\{\tau=\gamma\}}\right]\right\}
\end{aligned}
$$

for all $x \in \mathbf{R}_{+}$. This computation proves the claimed result for the lower value $\underline{V}_{1}$. The result for the upper value $\bar{V}_{1}$ is proved completely similarly.

In Proposition 3.2 we showed that the random horizon game can be transformed into an equivalent adjusted perpetual game. Moreover, we observe that the form the value function (3.6) associated with constant threshold policy is consistent with Proposition 3.2. It is also worth mentioning that the buyer follows actually a stopping rule "Stop at time $\tau \wedge T$ " which results into the payoff $g_{1}\left(X_{\tau}\right) \mathbf{1}_{\{\tau<T\}}+g_{1}^{+}\left(X_{T}\right) \mathbf{1}_{\{\tau \geq T\}}$. This property was used in (3.7).

3.2. Necessary Conditions. Having the expression (3.6) at hand, we proceed with the derivation of necessary conditions. Define the function $Q_{1}: \mathbf{R}_{+}^{3} \rightarrow \mathbf{R}_{+}$as

$$
\begin{aligned}
Q_{1}(x, z, y) & \left.=\mathbf{E}_{x}\left\{e^{-(r+\lambda)\left(\tau_{y} \wedge \gamma_{z}\right)}\left[\hat{g}_{1}\left(X_{\tau_{y}}\right) \mathbf{1}_{\left\{\tau_{y}<\gamma_{z}\right\}}+\hat{g}_{2}\left(X_{\gamma_{z}}\right)\left(X_{\gamma_{z}}\right)\right) \mathbf{1}_{\left\{\tau_{y}>\gamma_{z}\right\}}\right]\right\} \\
& =h_{1}(z, y) \psi_{r+\lambda}(x)+h_{2}(z, y) \varphi_{r+\lambda}(x)
\end{aligned}
$$


recall the definition of the functions $\hat{g}_{i}$ and $h_{i}, i=1,2$, from (3.2) and (3.4), respectively (see [1], expression (15)). Function $Q_{1}$ is the value function associated to the strategy constituted by the first hitting times $\tau_{y}$ and $\gamma_{z}$ to arbitrary boundaries $y$ and $z$ satisfying $z<y$. We assume now that the thresholds $z_{1}^{*}$ and $y_{1}^{*}$ give rise to an extremal expression for $Q_{1}$ in the sense that for all fixed (initial) state $x$, the point $\left(z_{1}^{*}, y_{1}^{*}\right)$ is a saddle point for the surface $(z, y) \mapsto Q_{1}(x, z, y)$. In other words, given the family of surfaces $(z, y) \mapsto Q_{1}(x, z, y)$, indexed by the initial states $x$, we assume that the point $\left(z_{1}^{*}, y_{1}^{*}\right)$ is saddle point for all of these surfaces. To determine first order necessary conditions for the saddle point, denote as $x_{o}$ the unique point satisfying the condition $\psi_{r+\lambda}\left(x_{o}\right)=\varphi_{r+\lambda}\left(x_{o}\right)$. Now, the conditions $\frac{\partial Q_{1}}{\partial z}\left(x_{o}, z_{1}^{*}, y_{1}^{*}\right)=\frac{\partial Q_{1}}{\partial y}\left(x_{o}, z_{1}^{*}, y_{1}^{*}\right)=0$ result into

$$
\left\{\begin{array}{l}
\frac{\partial h_{1}}{\partial z}\left(z_{1}^{*}, y_{1}^{*}\right)+\frac{\partial h_{2}}{\partial z}\left(z_{1}^{*}, y_{1}^{*}\right)=0 \\
\frac{\partial h_{1}}{\partial y}\left(z_{1}^{*}, y_{1}^{*}\right)+\frac{\partial h_{2}}{\partial y}\left(z_{1}^{*}, y_{1}^{*}\right)=0
\end{array}\right.
$$

Using the notation from (3.1), we find after differentiation and some elementary manipulations that the conditions (3.8) can be rewritten as

$$
\left\{\begin{array}{l}
\left(L_{\varphi}^{r+\lambda} \hat{g}_{2}\right)\left(z_{1}^{*}\right) \psi_{r+\lambda}\left(y_{1}^{*}\right)-\left(L_{\psi}^{r+\lambda} \hat{g}_{2}\right)\left(z_{1}^{*}\right) \varphi_{r+\lambda}\left(y_{1}^{*}\right)=B_{r+\lambda} \hat{g}_{1}\left(y_{1}^{*}\right), \\
\left(L_{\varphi}^{r+\lambda} \hat{g}_{1}\right)\left(y_{1}^{*}\right) \psi_{r+\lambda}\left(z_{1}^{*}\right)-\left(L_{\psi}^{r+\lambda} \hat{g}_{1}\right)\left(y_{1}^{*}\right) \varphi_{r+\lambda}\left(z_{1}^{*}\right)=B_{r+\lambda} \hat{g}_{2}\left(z_{1}^{*}\right),
\end{array}\right.
$$

Following [1], Lemma 4.1, we readily verify that the conditions (3.9) can be expressed as

$$
\left\{\begin{array}{l}
\int_{z_{1}^{*}}^{y_{1}^{*}}\left(\mathcal{G}_{r+\lambda} \hat{g}_{2}\right)(t)\left(\varphi_{r+\lambda}(t)-\frac{\varphi_{r+\lambda}\left(y_{1}^{*}\right)}{\psi_{r+\lambda}\left(y_{1}^{*}\right)} \psi_{r+\lambda}(t)\right) m^{\prime}(t) d t=\frac{B}{\psi_{r+\lambda}\left(y_{1}^{*}\right)}\left(\hat{g}_{2}\left(y_{1}^{*}\right)-\hat{g}_{1}\left(y_{1}^{*}\right)\right), \\
\int_{z_{1}^{*}}^{y_{1}^{*}}\left(\mathcal{G}_{r+\lambda} \hat{g}_{1}\right)(t)\left(\psi_{r+\lambda}(t)-\frac{\psi_{r+\lambda}\left(z_{1}^{*}\right)}{\varphi_{r+\lambda}\left(z_{1}^{*}\right)} \varphi_{r+\lambda}(t)\right) m^{\prime}(t) d t=\frac{B}{\varphi_{r+\lambda}\left(z_{1}^{*}\right)}\left(\hat{g}_{1}\left(z_{1}^{*}\right)-\hat{g}_{2}\left(z_{1}^{*}\right)\right) .
\end{array}\right.
$$

Denote now the candidate

$$
G_{1}(x)= \begin{cases}g_{1}(x), & x \geq y_{1}^{*}, \\ \lambda\left(R_{r+\lambda} g_{1}^{+}\right)(x)+Q_{1}\left(x, z_{1}^{*}, y_{1}^{*}\right), & x \in\left(z_{1}^{*}, y_{1}^{*}\right), \\ g_{2}(x), & x \leq z_{1}^{*} .\end{cases}
$$

Finally, the $r$-harmonicity of the candidate $G_{1}$ on the continuation region $\left(z_{1}^{*}, y_{1}^{*}\right)$ implies that the necessary conditions (3.8) can be rewritten as

$$
\left\{\begin{array}{l}
\left(L_{\varphi}^{r+\lambda} \hat{g}_{2}\right)\left(z_{1}^{*}\right)-\left(L_{\varphi}^{r+\lambda} \hat{g}_{1}\right)\left(y_{1}^{*}\right)=0 \\
\left(L_{\psi}^{r+\lambda} \hat{g}_{2}\right)\left(z_{1}^{*}\right)-\left(L_{\psi}^{r+\lambda} \hat{g}_{1}\right)\left(y_{1}^{*}\right)=0
\end{array}\right.
$$


see also [1], Corollary 4.2 .

The next proposition contains our main result on the necessary conditions for the optimal solution for Game 1.

Proposition 3.3. Assume that there is a pair $\left(z_{1}^{*}, y_{1}^{*}\right)$ satisfying the conditions (3.12) and that there exist thresholds $\hat{x}_{i}, i=1,2$, such that

$$
\mathcal{G}_{r+\lambda} \hat{g}_{i}(x) \gtreqless 0, \text { whenever } x \lesseqgtr \hat{x}_{i} \text {. }
$$

Then the pair $\left(z_{1}^{*}, y_{1}^{*}\right)$ is unique and $z_{1}^{*}<\hat{x}_{2}$ and $\hat{x}_{1}<y_{1}^{*}$. Moreover the value of Game 1 reads as $V_{1}(x)=$ $G_{1}(x)$ for all $x \in \mathbf{R}_{+}$, where $G_{1}$ is defined in (3.11).

Proof. We know from [1], Theorem 4.3 that under assumption (3.13) a pair satisfying (3.12) is necessary unique and that $z_{1}^{*}<\hat{x}_{2}$ and $\hat{x}_{1}<y_{1}^{*}$. To prove that the value of the game reads as (3.11), we follow the lines of the proof of Theorem 4.3 in [1]. First, assume that $x \in\left(z_{1}^{*}, y_{1}^{*}\right)$ and define the functionals

$$
\begin{aligned}
\Delta_{1}(x) & =\lambda\left(R_{r+\lambda} g_{1}^{+}\right)(x)+h_{1}\left(z_{1}^{*}, y_{1}^{*}\right) \psi_{r+\lambda}(x)+h_{2}\left(z_{1}^{*}, y_{1}^{*}\right) \varphi_{r+\lambda}(x)-g_{1}(x) \\
& =\hat{g}_{2}\left(z_{1}^{*}\right) \frac{\hat{\varphi}_{y_{1}^{*}}(x)}{\hat{\varphi}_{y_{1}^{*}}\left(z_{1}^{*}\right)}+\hat{g}_{1}\left(y_{1}^{*}\right) \frac{\hat{\psi}_{z_{1}^{*}}(x)}{\hat{\psi}_{z_{1}^{*}}\left(y_{1}^{*}\right)}-\hat{g}_{1}(x) \\
\Delta_{2}(x) & =\lambda\left(R_{r+\lambda} g_{1}^{+}\right)(x)+h_{1}\left(z_{1}^{*}, y_{1}^{*}\right) \psi_{r+\lambda}(x)+h_{2}\left(z_{1}^{*}, y_{1}^{*}\right) \varphi_{r+\lambda}(x)-g_{2}(x) \\
& =\hat{g}_{2}\left(z_{1}^{*}\right) \frac{\hat{\varphi}_{y_{1}^{*}}(x)}{\hat{\varphi}_{y_{1}^{*}}\left(z_{1}^{*}\right)}+\hat{g}_{1}\left(y_{1}^{*}\right) \frac{\hat{\psi}_{z_{1}^{*}}(x)}{\hat{\psi}_{z_{1}^{*}}\left(y_{1}^{*}\right)}-\hat{g}_{2}(x),
\end{aligned}
$$

where

$$
\begin{aligned}
& \hat{\varphi}_{y}(x)=\varphi_{r+\lambda}(x)-\frac{\varphi_{r+\lambda}(y)}{\psi_{r+\lambda}(y)} \psi_{r+\lambda}(x), \\
& \hat{\psi}_{z}(x)=\psi_{r+\lambda}(x)-\frac{\psi_{r+\lambda}(z)}{\varphi_{r+\lambda}(z)} \varphi_{r+\lambda}(x) .
\end{aligned}
$$

The saddle point condition (3.8) implies that the candidate $G_{1}$ is once continuously differentiable in $\mathbf{R}_{+}$and, consequently, that $\Delta_{1}\left(y_{1}^{*}\right)=\Delta_{1}^{\prime}\left(y_{1}^{*}\right)=0=\Delta_{2}\left(z_{1}^{*}\right)=\Delta_{2}^{\prime}\left(z_{1}^{*}\right)$. By standard differentiation we find that

$$
\begin{aligned}
& \frac{d}{d x}\left[\frac{\Delta_{1}(x)}{\hat{\psi}_{z_{1}^{*}}(x)}\right]=\frac{S^{\prime}(x)}{\hat{\psi}_{z_{1}^{*}}^{2}(x)}\left[\frac{-B \hat{g}_{2}\left(z_{1}^{*}\right)}{\varphi_{r+\lambda}\left(z_{1}^{*}\right)}-\frac{\hat{g}_{1}^{\prime}(x)}{S^{\prime}(x)} \hat{\psi}_{z_{1}^{*}}(x)+\frac{\hat{\psi}_{z_{1}^{*}}^{\prime}(x)}{S^{\prime}(x)} \hat{g}_{1}(x)\right], \\
& \frac{d}{d x}\left[\frac{\Delta_{2}(x)}{\hat{\varphi}_{y_{1}^{*}}(x)}\right]=\frac{S^{\prime}(x)}{\hat{\varphi}_{y_{1}^{*}}^{2}(x)}\left[\frac{B \hat{g}_{1}\left(y_{1}^{*}\right)}{\psi_{r+\lambda}\left(y_{1}^{*}\right)}-\frac{\hat{g}_{2}^{\prime}(x)}{S^{\prime}(x)} \hat{\varphi}_{y_{1}^{*}}(x)+\frac{\hat{\varphi}_{y_{1}^{*}}^{\prime}(x)}{S^{\prime}(x)} \hat{g}_{2}(x)\right],
\end{aligned}
$$


which imply together with (3.10) that

$$
\begin{aligned}
& \frac{d}{d x}\left[\frac{\Delta_{1}(x)}{\hat{\psi}_{z_{1}^{*}}(x)}\right]=\frac{S^{\prime}(x)}{\hat{\psi}_{z_{1}^{*}}^{2}(x)} \int_{x}^{y_{1}^{*}} \hat{\psi}_{z_{1}^{*}}(t)\left(\mathcal{G}_{r+\lambda} \hat{g}_{1}\right)(t) m^{\prime}(t) d t<0, \\
& \frac{d}{d x}\left[\frac{\Delta_{2}(x)}{\hat{\varphi}_{y_{1}^{*}}(x)}\right]=-\frac{S^{\prime}(x)}{\hat{\varphi}_{y_{1}^{*}}^{2}(x)} \int_{z_{1}^{*}}^{x} \hat{\varphi}_{y_{1}^{*}}(t)\left(\mathcal{G}_{r+\lambda} \hat{g}_{2}\right)(t) m^{\prime}(t) d t<0 .
\end{aligned}
$$

To see that the inequalities in (3.14) hold, we observe first using $(3.10)$ that $\left(\frac{\Delta_{1}}{\hat{\psi}_{z_{1}^{*}}}\right)^{\prime}\left(z_{1}^{*}\right)<0$ and $\left(\frac{\Delta_{2}}{\hat{\varphi}_{y_{1}^{*}}}\right)^{\prime}\left(y_{1}^{*}\right)<$ 0 . Moreover, since $0<z_{1}^{*}<\hat{x}_{2}$ and $\hat{x}_{1}<y_{1}^{*}<\infty$, where $\hat{x}_{2}$ and $\hat{x}_{1}$ are given in (3.13), and $x \in\left(z_{1}^{*}, y_{1}^{*}\right)$, the examination of the derivatives of the integrals reveals that the inequalities in (3.14) hold.

To conclude, we observe first that the condition (3.14) implies that $\frac{\Delta_{1}(x)}{\hat{\psi}_{z_{1}^{*}}^{*}(x)} \geq \frac{\Delta_{1}\left(y_{1}^{*}\right)}{\hat{\psi}_{z_{1}^{*}}^{*}\left(y_{1}^{*}\right)}=0$. Furthermore, since $\hat{\psi}_{z_{1}^{*}}(x)>0$, we find that $\Delta_{1}(x) \geq 0$ for all $x \in\left(z_{1}^{*}, y_{1}^{*}\right)$. Similarly we find that $\Delta_{2}(x) \leq 0$ for all $x \in\left(z_{1}^{*}, y_{1}^{*}\right)$. These properties of $\Delta_{i}$ imply that $g_{1}(x) \leq G_{1}(x) \leq g_{2}(x)$ for all $x \in\left(z_{1}^{*}, y_{1}^{*}\right)$. Moreover, since $z_{1}^{*}<\hat{x}_{2}$ and $y_{1}^{*}>\hat{x}_{1}$, we observe that $G_{1}$ is $r$-superharmonic on $\left(z_{1}^{*}, \infty\right)$ where the value is strictly smaller than $g_{2}(x)$ and $r$-subharmonic on $\left(0, y_{1}^{*}\right)$ where the value is strictly larger than $g_{1}(x)$. Thus the thresholds $z_{1}^{*}$ and $y_{1}^{*}$ give rise to a unique saddle point strategy and the value $V_{1}(x)=G_{1}(x)$ for all $x \in \mathbf{R}_{+}$.

In Proposition 3.3 we showed that given the additional condition (3.13), a solution of the pair (3.12) is necessarily unique. From a practical point of view this is a convenient result. Indeed, if we attempt to solve the pair (3.12) numerically for a particular example and our scheme converges to a solution, we can be sure that it is the unique optimal one. The condition (3.13) was needed in the proof of Proposition 3.3 to assure that functionals $L^{r+\lambda} \hat{g}_{i}$ behave nicely enough for the uniqueness result to hold - remember that $\left(L^{r+\lambda} \hat{g}_{i}\right)^{\prime}(x) \propto\left(\mathcal{G}_{r+\lambda} \hat{g}_{i}\right)(x)$. We propose in the next lemma a set of sufficient conditions for the assumption (3.13).

Lemma 3.4. Assume that there are thresholds $\tilde{x}_{i}, i=1,2$, such that

$$
\mathcal{G}_{r} g_{i}(x) \gtreqless 0, \text { whenever } x \lesseqgtr \tilde{x}_{i} \text {. }
$$

In addition, assume that

- $g_{1}(x) \geq 0$ for all $x>0$ or that $\mathcal{G}_{r} g_{1}$ is non-increasing, and

- $\left(g_{1}^{+}-g_{2}\right)$ and $\mathcal{G}_{r} g_{2}$ are non-increasing.

Then the condition (3.13) holds. 
Proof. The result follows from the expressions $\mathcal{G}_{r+\lambda} \hat{g}_{1}=\mathcal{G}_{r} g_{1}+\lambda\left(g_{1}^{+}-g_{1}\right)$, where $g_{1}^{+}-g_{1}$ is non-increasing and $\mathcal{G}_{r+\lambda} \hat{g}_{2}=\mathcal{G}_{r} g_{2}+\lambda\left(g_{1}^{+}-g_{2}\right)$.

We note also from Proposition 3.3 that the stopping times $\tau_{y_{1}^{*}}$ and $\gamma_{z_{1}^{*}}$ do not tell the entire story about the optimal stopping rules. Indeed, the optimal stopping rule for the issuer is "stop at time $\gamma_{z_{1}^{*}}=\inf \{t \geq 0 \mid$ $\left.X_{t} \leq z_{1}^{*}\right\}$ ", but for the buyer optimal rule is "stop at time $\tau_{y_{1}^{*}}=\inf \left\{t \geq 0 \mid X_{t} \geq y_{1}^{*}\right\}$, but if $T<\tau_{y_{1}^{*}}$, stop at time $T$ whenever $g_{1}\left(X_{T}\right)>0$ " so that the optimal rule for the buyer is not pure threshold rule.

While Proposition 3.3 catches a relatively large range of problems, our assumptions are not usually satisfied if exercise payoffs have option characteristics - for example if $g_{i}(x)=\left(x-c_{i}\right)^{+}$, where $c_{1}>c_{2}>0$. In the next result we propose a set of necessary conditions for a class of problems of this kind.

Corollary 3.5. Assume that there exists $\bar{x}_{i}<\hat{x}_{i}$ so that $g_{i}(x)=0$ on $\left(0, \bar{x}_{i}\right),\left(\mathcal{C}_{r+\lambda} \hat{g}_{i}\right)>0$ on $\left(\bar{x}_{i}, \hat{x}_{i}\right)$, and $\left(\mathcal{C}_{r+\lambda} \hat{g}_{i}\right)<0$ on $\left(\hat{x}_{i}, \infty\right), i=1,2$. Assume also that the threshold

$$
\hat{y}_{\bar{x}_{2}}^{*}=\underset{y}{\operatorname{argmax}}\left\{\frac{\hat{g}_{1}(y)}{\psi_{r+\lambda}(y)-\frac{\psi_{r+\lambda}\left(\bar{x}_{2}\right)}{\varphi_{r+\lambda}\left(\bar{x}_{2}\right)} \varphi_{r+\lambda}(y)}\right\}
$$

exists. If there exists a pair $\left(z_{1}^{*}, y_{1}^{*}\right) \in\left(\bar{x}_{2}, \hat{x}_{2}\right) \times\left(\hat{x}_{1}, \hat{y}_{\bar{x}_{2}}^{*}\right)$ satisfying the first order conditions (3.12), then the conclusion of Proposition 3.3 are satisfied and the value of the game reads as in (3.11).

Proof. The result follows from Proposition 3.3 after noticing that $\hat{y}_{\bar{x}_{2}}^{*}$ is the corner solution to the lower equation of (3.9).

If there does not exist an internal solution, then the pair $\left(\bar{x}_{2}, \hat{y}_{\bar{x}_{2}}^{*}\right)$ constitutes a corner solution, which is the saddle point solution and the solution reads as

$$
V_{1}(x)= \begin{cases}g_{1}(x), & \text { if } x \geq \hat{y}_{\bar{x}_{2}}^{*} \\ \lambda\left(R_{r+\lambda} g_{1}^{+}\right)(x)+\frac{\psi_{r+\lambda}\left(\bar{x}_{2}\right) \varphi_{r+\lambda}(x)-\varphi_{r+\lambda}\left(\bar{x}_{2}\right) \psi_{r+\lambda}(x)}{\varphi_{r+\lambda}\left(\hat{y}_{\bar{x}_{2}}^{*}\right) \psi_{r+\lambda}\left(\bar{x}_{2}\right)-\varphi_{r+\lambda}\left(\bar{x}_{2}\right) \psi_{r+\lambda}\left(\hat{y}_{\bar{x}_{2}}^{*}\right)} \hat{g}_{1}\left(\hat{y}_{\bar{x}_{2}}^{*}\right), & \text { if } x \in\left(\bar{x}_{2}, \hat{y}_{\bar{x}}^{*}\right) \\ 0, & \text { if } x \leq \bar{x}_{2} .\end{cases}
$$

3.3. Sufficient Conditions. The main objective of this section is to propose a set of sufficient conditions for the solvability of the game. To this end, we prove first the following lemma.

Lemma 3.6. Let $b \in \mathbf{R}_{+}$. Then $\frac{\psi_{r}(x)}{\psi_{r}(b)}>\frac{\psi_{r+\lambda}(x)}{\psi_{r+\lambda}(b)}$ for all $x<b$ and the function $x \mapsto \frac{\psi_{r+\lambda}(x)}{\psi_{r}(x)}$ is monotonically increasing. 
Proof. Let $x<b<\infty$. From [3], p. 18, we have $\mathbf{E}_{x}\left\{e^{-r \tau_{b}}\right\}=\frac{\psi_{r}(x)}{\psi_{r}(b)}$, where $\tau_{b}=\inf \left\{t \geq 0 \mid X_{t}=b\right\}$. Then

$$
\frac{\psi_{r}(x)}{\psi_{r}(b)}=\mathbf{E}_{x}\left\{e^{-r \tau_{b}}\right\}>\mathbf{E}_{x}\left\{e^{-(r+\lambda) \tau_{b}}\right\}=\frac{\psi_{r+\lambda}(x)}{\psi_{r+\lambda}(b)}
$$

From this we also see that $\frac{\psi_{r+\lambda}}{\psi_{r}}$ is monotonically increasing.

The next theorem, which is the main result of this subsection, gives a set of conditions under which the optimal solution for Game 1 is given by (3.12) and (3.11).

Theorem 3.7. Assume that the boundaries 0 and $\infty$ are natural for the underlying $X$, that condition (3.13) holds, and that for $i=1,2$,

(1) $\mathcal{G}_{r} g_{i} \in \mathcal{L}_{1}^{r}$,

(2) $\lim _{x \rightarrow \infty}\left|\frac{g_{i}(x)}{\psi_{r}(x)}\right|=0$,

(3) $\mathcal{G}_{r} g_{1}(x)>\mathcal{G}_{r} g_{2}(x)$ for all $x \in \mathbf{R}_{+} \backslash D$.

Then there exist a unique pair $\left(z_{1}^{*}, y_{1}^{*}\right)$ satisfying the first order conditions (3.12) and the value $V_{1}$ of Game 1 reads as in (3.11).

Proof. First, we find by coupling the assumption (3) with the inequality $g_{2} \geq g_{1}$ that

$$
\left(\mathcal{G}_{r+\lambda} \hat{g}_{1}\right)(x)=\left(\mathcal{G}_{r} g_{1}\right)(x)+\lambda\left(g_{1}^{+}(x)-g_{1}(x)\right)>\left(\mathcal{G}_{r} g_{2}\right)(x)+\lambda\left(g_{1}^{+}(x)-g_{2}(x)\right)=\left(\mathcal{G}_{r+\lambda} \hat{g}_{2}\right)(x),
$$

for all $x \in \mathbf{R}_{+} \backslash D$. Furthermore, since the functions $g_{i} \in \mathcal{L}_{1}^{r}$, the assumption (1) implies that

$$
\mathcal{G}_{r+\lambda} \hat{g}_{i}=\mathcal{G}_{r} g_{i}+\lambda\left(g_{1}^{+}-g_{i}\right) \in \mathcal{L}_{1}^{r+\lambda},
$$

for $i=1,2$. Our next objective is to show that

$$
\lim _{x \rightarrow \infty}\left(L_{\varphi}^{r+\lambda} \hat{g}_{i}\right)(x)=\lim _{x \rightarrow 0}\left(L_{\psi}^{r+\lambda} \hat{g}_{i}\right)(x)=0 .
$$

To this end, let $b \in \mathbf{R}_{+}$. Since the function $\frac{\psi_{r}(x)}{\psi_{r+\lambda}(x)}$ is decreasing, see Lemma 3.6, we find

$$
0 \leq \lim _{x \rightarrow \infty}\left|\frac{\hat{g}_{i}(x)}{\psi_{r+\lambda}(x)}\right| \leq \frac{\psi_{r}(b)}{\psi_{r+\lambda}(b)} \lim _{x \rightarrow \infty}\left|\frac{g_{i}(x)-\lambda\left(R_{r+\lambda} g_{1}^{+}\right)(x)}{\psi_{r}(x)}\right|=0,
$$

for $i=1,2$. Here, the last inequality follows from the assumption (2) and Proposition 4 from [11]. By coupling (3.18) with (2.1) and (3.16), we find that

$$
\left(L_{\varphi}^{r+\lambda} \hat{g}_{i}\right)(x)=-\int_{x}^{\infty} \varphi_{r+\lambda}(y)\left(\mathcal{G}_{r+\lambda} \hat{g}_{i}\right)(y) m^{\prime}(y) d y \rightarrow 0, \text { as } x \rightarrow \infty
$$


where the integral representation follows from [1], Corollary 3.2. In addition, since $g_{1}$ and $g_{2}$ are bounded from below, Corollary 3.2 from [1] implies that

$$
\left(L_{\psi}^{r+\lambda} \hat{g}_{i}\right)(x)=\int_{0}^{x} \psi_{r+\lambda}(y)\left(\mathcal{G}_{r+\lambda} \hat{g}_{i}\right)(y) m^{\prime}(y) d y \rightarrow 0, \text { as } x \rightarrow 0 .
$$

Thus we have established the condition (3.17). Now, the conditions (3.13) and (3.15) - (3.17) guarantee that the claimed result follows from [1], Theorem 4.4.

Theorem 3.7 states a set of conditions under which a unique pair $\left(z_{1}^{*}, y_{1}^{*}\right)$ satisfying the first order conditions (3.12) exists and under which the value of the Game 1 can be written as (3.11). We remark that these conditions do not depend on the jump rate $\lambda$. Furthermore, we know from Lemma 3.4 that the condition (3.13) can be substituted with a set of conditions that are also independent of $\lambda$. Thus, when using our results to check whether a particular example of Game 1 has a (unique) solution, the value of $\lambda$ does not play any role.

\section{Game 2}

4.1. Equivalent formulation of the game. This section is devoted to the study of the solvability of Game 2. The analysis is completely analogous to the Section 3. Again, we begin with the ansatz that the game has a saddle point equilibrium and that the continuation region $\left(z_{2}^{*}, y_{2}^{*}\right) \subset \mathbf{R}_{+}$has compact closure. Now, because the terminal date $T$ is observable to the issuer and she knows that after that time the buyer cannot exercise, it is clear that she will exercise at time $T$ if and only if $g_{2}\left(X_{T}\right)<0$. Thus, in an infinitesimal time interval $d t$, the Poisson process jumps with probability $\lambda d t$ leaving the buyer with payoff $g_{2}^{-}(x)=\min \left\{g_{2}(x), 0\right\}$. With probability $1-\lambda d t$ there is no jump which results in additional expected present value. Analogously to Game 1 , we deduce that the candidate $G_{2}$ must satisfy the condition $\mathcal{G}_{r+\lambda} G_{2}(x)=-\lambda g_{2}^{-}(x)$ for all $x \in\left(z_{2}^{*}, y_{2}^{*}\right)$ and, consequently, the candidate can be represented as

$$
G_{2}(x)=\mathbf{E}_{x}\left\{\lambda \int_{0}^{\tau_{y_{2}^{*}} \wedge \gamma_{z_{2}^{*}}} e^{-(r+\lambda) s} g_{2}^{-}\left(X_{s}\right) d s+e^{-(r+\lambda)\left(\tau_{y_{2}^{*}} \wedge \gamma_{z_{2}^{*}}\right)}\left[g_{1}\left(X_{\left.\tau_{y_{2}^{*}}\right)} \mathbf{1}_{\left\{\tau_{\left.y_{2}^{*}<\gamma_{2}^{*}\right\}}\right.}+g_{2}\left(X_{\gamma_{z_{2}^{*}}^{*}}\right) \mathbf{1}_{\left\{\tau_{y_{2}^{*}}>\gamma_{z_{2}^{*}}\right\}}\right]\right\}\right.
$$

for all $x \in \mathbf{R}_{+}$. As in Game 1, this form is the correct form of the value function for the associated perpetual game.

Proposition 4.1. The upper and lower values can for Game 2 be rewritten as

$$
\bar{V}_{2}(x)=\inf _{\gamma \in \mathcal{T}_{0}} \sup _{\tau \in \mathcal{T}_{0}} \tilde{\Pi}_{2}(x, \tau, \gamma), \underline{V}_{2}(x)=\sup _{\tau \in \mathcal{T}_{0}} \inf _{\gamma \in \mathcal{T}_{0}} \tilde{\Pi}_{2}(x, \tau, \gamma)
$$


where

$\tilde{\Pi}_{2}(x, \tau, \gamma)=\mathbf{E}_{x}\left\{\lambda \int_{0}^{\tau \wedge \gamma} e^{-(r+\lambda) s} g_{2}^{-}\left(X_{s}\right) d s+e^{-(r+\lambda)(\tau \wedge \gamma)}\left[g_{1}\left(X_{\tau}\right) \mathbf{1}_{\{\tau<\gamma\}}+g_{2}\left(X_{\gamma}\right) \mathbf{1}_{\{\tau>\gamma\}}+g_{3}\left(X_{\gamma}\right) \mathbf{1}_{\{\tau=\gamma\}}\right]\right\}$ for all $x \in \mathbf{R}_{+}$.

Proof. Completely similar to the proof of Proposition 3.2.

Similarly to Game 1, we remark that the issuer follows now a stopping rule "Stop at time $\gamma \wedge T$ " which results into the payoff $g_{2}\left(X_{\gamma}\right) \mathbf{1}_{\{\gamma<T\}}+g_{2}^{-}\left(X_{T}\right) \mathbf{1}_{\{\gamma \geq T\}}$.

4.2. Necessary conditions. In order to simplify the notations, we denote

$$
\left\{\begin{array}{l}
\check{g}_{1}(x)=g_{1}(x)-\lambda\left(R_{r+\lambda} g_{2}^{-}\right)(x), \\
\check{g}_{2}(x)=g_{2}(x)-\lambda\left(R_{r+\lambda} g_{2}^{-}\right)(x) .
\end{array}\right.
$$

Moreover define the function $Q_{2}: \mathbf{R}_{+}^{3} \rightarrow \mathbf{R}_{+}$as

$$
\begin{aligned}
Q_{2}(x, z, y) & =\mathbf{E}_{x}\left\{e^{-(r+\lambda)\left(\tau_{y} \wedge \gamma_{z}\right)}\left[\left(\check{g}_{1}\left(X_{\tau_{y}}\right) \mathbf{1}_{\left\{\tau_{y}<\gamma_{z}\right\}}+\check{g}_{2}\left(X_{\gamma_{z}}\right)\left(X_{\gamma_{z}}\right)\right) \mathbf{1}_{\left\{\tau_{y}>\gamma_{z}\right\}}\right]\right\} \\
& =k_{1}(z, y) \psi_{r+\lambda}(x)+k_{2}(z, y) \varphi_{r+\lambda}(x),
\end{aligned}
$$

where the functions $k_{1}: \mathbf{R}_{+}^{2} \rightarrow \mathbf{R}$ are defined as

$$
\left\{\begin{array}{l}
k_{1}(z, y)=\frac{\varphi_{r+\lambda}(y) \check{g}_{2}(z)-\varphi_{r+\lambda}(z) \check{g}_{1}(y)}{\varphi_{r+\lambda}(y) \psi_{r+\lambda}(z)-\varphi_{r+\lambda}(z) \psi_{r+\lambda}(y)} \\
k_{2}(z, y)=\frac{\psi_{r+\lambda}(z) \check{g}_{1}(y)-\psi_{r+\lambda}(y) \check{g}_{2}(z)}{\varphi_{r+\lambda}(y) \psi_{r+\lambda}(z)-\varphi_{r+\lambda}(z) \psi_{r+\lambda}(y)}
\end{array}\right.
$$

Analogously to Section 3, we assume that for every fixed $x$, the $(z, y) \rightarrow Q_{2}(x, z, y)$ has a unique saddle point $\left(z_{2}^{*}, y_{2}^{*}\right)$, which does note depend on $x$. Then the first order necessary conditions for this saddle point can be written as

$$
\left\{\begin{array}{l}
\left(L_{\varphi}^{r+\lambda} \check{g}_{2}\right)\left(z_{2}^{*}\right)-\left(L_{\varphi}^{r+\lambda} \check{g}_{1}\right)\left(y_{2}^{*}\right)=0, \\
\left(L_{\psi}^{r+\lambda} \check{g}_{2}\right)\left(z_{2}^{*}\right)-\left(L_{\psi}^{r+\lambda} \check{g}_{1}\right)\left(y_{2}^{*}\right)=0 .
\end{array}\right.
$$

The next proposition contains our main result on the necessary conditions for the optimal solution for Game 2 . 
Proposition 4.2. Assume that there is a pair $\left(z_{2}^{*}, y_{2}^{*}\right)$ satisfying the conditions (4.4) and that there are thresholds $\check{x}_{i}, i=1,2$, such that

$$
\mathcal{G}_{r+\lambda} \check{g}_{i}(x) \gtreqless 0, \text { whenever } x \lesseqgtr \check{x}_{i} \text {. }
$$

Then the pair $\left(z_{2}^{*}, y_{2}^{*}\right)$ is unique and $z_{2}^{*} \leq z_{1}^{*}$ and $y_{2}^{*} \leq y_{1}^{*}$. Furthermore the value $V_{2}$ of Game 2 reads as

$$
V_{2}(x)= \begin{cases}g_{1}(x), & x \geq y_{2}^{*}, \\ \lambda\left(R_{r+\lambda} g_{2}^{-}\right)(x)+k_{1}\left(z_{2}^{*}, y_{2}^{*}\right) \psi_{r+\lambda}(x)+k_{2}\left(z_{2}^{*}, y_{2}^{*}\right) \varphi_{r+\lambda}(x), & x \in\left(z_{2}^{*}, y_{2}^{*}\right), \\ g_{2}(x), & x \leq z_{2}^{*},\end{cases}
$$

where the functions $k_{i}, i=1,2$, are defined in (4.3).

Proof. Completely analogous to the proof of Proposition 3.3.

Similarly to Proposition 3.3, we posed in Proposition 4.2 the additional assumption (4.5) to assure that the functionals $L^{r+\lambda} \check{g}_{i}$ behave well enough so that the uniqueness of the solution is guaranteed. In this case, as in Game 1, we propose sufficient conditions to (4.5) which do not depend on $\lambda$. These conditions are listed in the next lemma.

Lemma 4.3. Assume that there are thresholds $\tilde{x}_{i}, i=1,2$, such that $\left(\mathcal{G}_{r}\right) g_{i}(x) \gtreqless 0$, whenever $x \lesseqgtr \tilde{x}_{i}$. In addition, assume that

- $\left(\mathcal{G}_{r} g_{1}\right)$ and $g_{2}^{-}-g_{1}$ are non-increasing, and

- $\left(\mathcal{G}_{r} g_{2}\right)$ is non-increasing or $g_{2} \leq 0$ for all $x>0$.

Then the condition (4.5) holds.

Proof. Similar to the proof of Lemma 3.4.

Similar to Proposition 3.3, the optimal thresholds times $\tau_{y_{2}^{*}}$ and $\gamma_{z_{2}^{*}}$ do not tell the whole truth about the optimal stopping times. The optimal stopping time for the issuer is now "stop at time $\gamma_{z_{2}^{*}}$, but if $T<\gamma_{z_{2}^{*}}$ and $g_{2}\left(X_{T}\right)<0$, stop at time $T$, else do not stop", whilst the optimal stopping time for the buyer is "stop at time $\tau_{y_{2}^{*}}=\inf \left\{t \geq 0 \mid X_{t} \geq y_{2}^{*}\right\} "$

Corollary 4.4. Assume that there exists $\bar{x}_{i}<\check{x}_{i}$ so that $g_{i}(x)=0$ on $\left(0, \bar{x}_{i}\right),\left(\mathcal{C}_{r+\lambda} \check{g}_{i}\right)>0$ on $\left(\bar{x}_{i}, \check{x}_{i}\right)$, and $\left(\mathcal{C}_{r+\lambda} \hat{g}_{i}\right)<0$ on $\left(\check{x}_{i}, \infty\right), i=1,2$. Assume also that the threshold

$$
\check{y}_{\bar{x}_{2}}^{*}=\underset{y}{\operatorname{argmax}}\left\{\frac{\check{g}_{1}(y)}{\psi_{r+\lambda}(y)-\frac{\psi_{r+\lambda}\left(\bar{x}_{2}\right)}{\varphi_{r+\lambda}\left(\bar{x}_{2}\right)} \varphi_{r+\lambda}(y)}\right\}
$$


exists. If there exists a pair $\left(z_{2}^{*}, y_{2}^{*}\right) \in\left(\bar{x}_{2}, \breve{x}_{2}\right) \times\left(\check{x}_{1}, \check{y}_{\bar{x}_{2}}^{*}\right)$ satisfying the first order conditions (4.4), then the conclusion of Proposition 4.2 are satisfied and the value of the game reads as in (4.6).

Proof. Proof is similar to that of Corollary 3.5.

If there does not exist an internal solution, then the pair $\left(\bar{x}_{2}, \check{y}_{\bar{x}_{2}}^{*}\right)$ constitutes a corner solution, which is the saddle point solution and the solution reads as

$$
V_{2}(x)= \begin{cases}g_{1}(x), & \text { if } x \geq \check{y}_{\bar{x}_{2}}^{*} \\ \lambda\left(R_{r+\lambda} g_{2}^{-}\right)(x)+\frac{\psi_{r+\lambda}\left(\bar{x}_{2}\right) \varphi_{r+\lambda}(x)-\varphi_{r+\lambda}\left(\bar{x}_{2}\right) \psi_{r+\lambda}(x)}{\varphi_{r+\lambda}\left(\check{y}_{\bar{x}}^{*}\right) \psi_{r+\lambda}\left(\bar{x}_{2}\right)-\varphi_{r+\lambda}\left(\bar{x}_{2}\right) \psi_{r+\lambda}\left(\check{y}_{\bar{x}_{2}}^{*}\right)} \check{g}_{1}\left(\check{y}_{\bar{x}_{2}}^{*}\right), & \text { if } x \in\left(\bar{x}_{2}, \check{y}_{\bar{x}_{2}}^{*}\right) \\ 0, & \text { if } x \leq \bar{x}_{2} .\end{cases}
$$

4.3. Sufficient conditions. The next theorem contains a set of sufficient conditions for the optimal solution for Game 2 .

Theorem 4.5. Assume that the boundaries 0 and $\infty$ are natural for the underlying $X$, that condition (4.5) hold, and that the conditions 1-3 in Theorem 3.7 holds for $i=1,2$. Then there exist a unique pair satisfying the first order conditions (4.4) and the value $V_{2}$ of Game 2 reads as in (4.6).

Proof. The proof is analogous to that of Theorem 3.7.

Theorem 4.5 states sufficient conditions under which an optimal pair $\left(z_{2}^{*}, y_{2}^{*}\right)$ uniquely exists and under which the value of Game 2 can be expressed as in (4.6). Using Lemma 4.3 the condition (4.5) can be expressed independent of $\lambda$. Therefore, similar to Game 1, we remark that with a particular example, the conditions of the theorem can be checked without any reference to the jump rate $\lambda$.

\section{COMPARISON AND ASYMPTOTICS}

In the previous sections we studied the solvability of Games 1 and 2. In particular, we derived necessary and sufficient conditions for the solutions to be given by (3.11) and (4.6). In this section, we study further the properties of these solutions. In particular, we are interested in finding orderings of the stopping thresholds and the value functions. Furthermore, we study the asymptotic behavior of the optimal characteristics with respect to jump rate $\lambda$. To this end, we define two more Dynkin games. First of these is the infinite horizon Dynkin game, which is defined using (1.2) and (2.3) in the absence of terminating event taking place at time $T$. For a comprehensive analysis of this game, see [1]. Denote the value of this game as $V$ and the optimal exercise thresholds as $\left(z^{*}, y^{*}\right)$. The second additional game is the game with random time horizon in the case where the terminating event is not observable to either of the players - we refer to this game as Game 3 . The 
upper and lower values of Game 3 are $\inf _{\gamma \in \mathcal{T}_{0}} \sup _{\tau \in \mathcal{T}_{0}} \tilde{\Pi}_{3}(x, \tau, \gamma)$ and $\sup _{\tau \in \mathcal{T}_{0}} \inf _{\gamma \in \mathcal{T}_{0}} \tilde{\Pi}_{3}(x, \tau, \gamma)$ respectively, where

$$
\tilde{\Pi}_{3}(x, \tau, \gamma)=\mathbf{E}_{x}\left\{e^{-(r+\lambda)(\tau \wedge \gamma)}\left[g_{1}\left(X_{\tau}\right) \mathbf{1}_{\{\tau<\gamma\}}+g_{2}\left(X_{\gamma}\right) \mathbf{1}_{\{\tau>\gamma\}}+g_{3}\left(X_{\gamma}\right) \mathbf{1}_{\{\tau=\gamma\}}\right]\right\} .
$$

In fact, Game 3 is an infinite horizon game with discount rate $r+\lambda$. Hence we know from [1] that under certain assumptions this game has a Nash equilibrium given by the unique thresholds $\left(z_{3}^{*}, y_{3}^{*}\right)$. We denote the value of this game as $V_{3}$. It is worth pointing out that Proposition 3.2 implies that if the function $g_{1}$ is nonpositive, the value of Game 1 coincides with the value of Game 3. Similarly, Proposition 4.1 implies that if the function $g_{2}$ is nonnegative, the value of Game 2 coincides with the value of Game 3.

5.1. Ordering of the thresholds and the values. The following proposition is our main result on the ordering of optimal characteristics of the games.

Proposition 5.1. (A) Assume that Game 1, Game 2 and Game 3 have unique saddle point solutions. Then the following orderings hold

- $V_{1}(x) \geq V_{3}(x) \geq V_{2}(x)$ everywhere.

- $z_{1}^{*} \geq z_{3}^{*} \geq z_{2}^{*}$ and $y_{1}^{*} \geq y_{3}^{*} \geq y_{2}^{*}$ always.

(B) If in addition the infinite horizon game has a unique saddle point solution and $g_{2}$ is non-negative, then

- $V(x) \geq V_{1}(x) \geq V_{3}(x) \geq V_{2}(x)$ for all $x \in \mathbf{R}_{+}$.

- $z^{*} \geq z_{1}^{*} \geq z_{3}^{*} \geq z_{2}^{*}$ and $y^{*} \geq y_{1}^{*} \geq y_{3}^{*} \geq y_{2}^{*}$.

Proof. (A) Let us first prove the orderings between Game 1 and Game 2. Recall the definitions of $\tilde{\Pi}_{1}(x, \tau, \gamma)$ and $\tilde{\Pi}_{2}(x, \tau, \gamma)$ from Propositions 3.2 and 4.1 respectively. Now

$$
\begin{aligned}
\tilde{\Pi}_{1}(x, \tau, \gamma) & =\mathbf{E}_{x}\left\{\lambda \int_{0}^{\tau \wedge \gamma} e^{-(r+\lambda) s} g_{1}^{+}\left(X_{s}\right) d s+e^{-(r+\lambda)(\tau \wedge \gamma)}\left[g_{1}\left(X_{\tau}\right) \mathbf{1}_{\{\tau<\gamma\}}+g_{2}\left(X_{\gamma}\right) \mathbf{1}_{\{\tau>\gamma\}}+g_{3}\left(X_{\gamma}\right) \mathbf{1}_{\{\tau=\gamma\}}\right]\right\} \\
& \geq \mathbf{E}_{x}\left\{\lambda \int_{0}^{\tau \wedge \gamma} e^{-(r+\lambda) s} g_{2}^{-}\left(X_{s}\right) d s+e^{-(r+\lambda)(\tau \wedge \gamma)}\left[g_{1}\left(X_{\tau}\right) \mathbf{1}_{\{\tau<\gamma\}}+g_{2}\left(X_{\gamma}\right) \mathbf{1}_{\{\tau>\gamma\}}+g_{3}\left(X_{\gamma}\right) \mathbf{1}_{\{\tau=\gamma\}}\right]\right\} \\
& =\tilde{\Pi}_{2}(x, \tau, \gamma),
\end{aligned}
$$

for all $x \in \mathbf{R}_{+}$and $\tau, \gamma \in \mathcal{T}_{0}$. Thus

$$
V_{1}(x)=\sup _{\tau \in \mathcal{T}_{0}} \inf _{\gamma \in \mathcal{T}_{0}} \tilde{\Pi}_{1}(x, \tau, \gamma) \geq \sup _{\tau \in \mathcal{T}_{0}} \inf _{\gamma \in \mathcal{T}_{0}} \tilde{\Pi}_{2}(x, \tau, \gamma)=V_{2}(x)
$$

Suppose now, contrary to our claim, that $y_{1}^{*}<y_{2}^{*}$ and let $x \in\left(y_{1}^{*}, y_{2}^{*}\right)$ so that $x$ is in the continuation region of Game 1, and in the stopping region of Game 2. Then $V_{1}(x)=g_{1}(x)<V_{2}(x)$, contrary to (5.1). The same reasoning applies to the case $z_{2}^{*} \leq z_{1}^{*}$. Next, recall the definition of $\tilde{\Pi}_{3}$ from beginning of the section. 
We see that $\tilde{\Pi}_{1} \geq \tilde{\Pi}_{3} \geq \tilde{\Pi}_{2}$ and using reasoning as above we find that $V_{1} \geq V_{3} \geq V_{2}$. The claimed inequalities for the thresholds follows as above.

(B) Let $g_{2}$ be non-negative and recall the definition of $\Pi(x, \tau, \gamma)$ from (1.2). We shall compare it to $\Pi_{1}$ from (2.4). We know that the value function satisfies $V(x)=\sup _{\tau \in \mathcal{T}_{0}} \inf _{\gamma \in \mathcal{T}_{0}} \Pi(x, \tau, \gamma)$ and similarly $V_{1}(x)=\sup _{\tau \in \mathcal{T}_{1}} \inf _{\gamma \in \mathcal{T}_{0}} \Pi_{1}(x, \tau, \gamma)$. Letting $\hat{\mathcal{T}}_{1}=\left\{\tau \in \mathcal{T}_{1} \mid \tau \leq T\right\}$ and $\hat{\mathcal{T}}_{1}^{c}=\mathcal{T}_{1} \backslash \hat{\mathcal{T}}_{1}$ we can write

$$
V_{1}(x)=\max \left\{\sup _{\tau \in \tilde{\mathcal{T}}_{1}} \inf _{\gamma \in \mathcal{T}_{0}} \Pi_{1}(x, \tau, \gamma) ; \sup _{\tau \in \tilde{\mathcal{T}}_{1}^{c}} \inf _{\gamma \in \mathcal{T}_{0}} \Pi_{1}(x, \tau, \gamma)\right\},
$$

the last term being zero due to non-negativeness of $g_{2}$. For the first term on the right hand side we get

$$
\sup _{\tau \in \hat{\mathcal{T}}_{1}} \inf _{\gamma \in \mathcal{T}_{0}} \Pi_{1}(x, \tau, \gamma)=\sup _{\tau \in \mathcal{T}_{1}} \inf _{\gamma \in \mathcal{T}_{0}} \Pi(x, \tau, \gamma)
$$

and again due to non-negativeness of $g_{2}$ for the second term we get the inequality

$$
\sup _{\tau \in \tilde{\mathcal{T}}_{1}^{c}} \inf _{\gamma \in \mathcal{T}_{0}} \Pi_{1}(x, \tau, \gamma)=0 \leq \inf _{\gamma \in \mathcal{T}_{0}} \Pi(x, \infty, \gamma) \leq \sup _{\tau \in \tilde{\mathcal{T}}_{1}^{c}} \inf _{\gamma \in \mathcal{T}_{0}} \Pi(x, \tau, \gamma)
$$

Substituting these to $(5.2)$ we get

$$
V_{1}(x) \leq \max \left\{\sup _{\tau \in \mathcal{\mathcal { T }}_{1}} \inf _{\gamma \in \hat{\mathcal{T}}_{0}} \Pi(x, \tau, \gamma) ; \sup _{\tau \in \mathcal{T}_{1}^{c}} \inf _{\gamma \in \mathcal{T}_{0}} \Pi(x, \tau, \gamma)\right\}=\sup _{\tau \in \mathcal{T}_{1}} \inf _{\gamma \in \mathcal{T}_{0}} \Pi(x, \tau, \gamma)=V(x),
$$

the first equality being true, since in the maximization we have two disjoint sets for which $\hat{\mathcal{T}}_{1} \cup \hat{\mathcal{T}}_{1}^{c}=\mathcal{T}_{1}$ and the last equality follows from the fact that $\mathcal{T}_{1}=\mathcal{T}_{0}$ in the absence of terminating event.

Suppose, contrary to our claim, that $y^{*}<y_{1}^{*}$ and let $x \in\left(y^{*}, y_{1}^{*}\right)$, so that $x$ is in the continuation region of stochastic time horizon case, and in the stopping region of infinite time horizon case. Then $V(x)=g_{1}(x)<$ $V_{1}(x)$, contrary to $(5.3)$. The same reasoning applies to the case $z_{1}^{*} \leq z^{*}$.

Intuitively, the item (A) of Proposition 5.1 is not surprising. Indeed, if the issuer has inside information about the terminating event, it will make the value of the game smaller as there is one additional stopping time in the set over which the issuer minimizes. Similarly, if the buyer has inside information about the terminating event, the value will be larger. In Game 3, the value is naturally in between these two extremes. Furthermore, the exercise thresholds are ordered as one could guess from orderings of the value functions, the principal idea being: The more you know, the longer you wait.

The item (B) is also intuitively quite clear. Since $g_{2} \geq 0$, there is no risk of ending up on trajectory leading inevitably into negative payoff. By coupling this with the fact that Game 1 will end in finite time almost surely, the ordering $V \geq V_{1}$ becomes evident as the less time to maximize the payoff which is bound 
to be nonnegative. We stress here that the positiveness of $g_{2}$ is indeed required for the inequalities $V \geq V_{1}$, $z^{*} \geq z_{1}^{*}$ and $y^{*} \geq y_{1}^{*}$ to hold. We will give a numerical example at the end of Section 6 where these inequalities are reversed for a function $g_{2}$ that takes also negative values.

5.2. Some asymptotics. In this subsection we study the limiting behavior of the optimal characteristics of Games 1 and 2 when the jump rate $\lambda$ tends to infinity as well as when it tends to zero. The next proposition is our main result on this matter.

Proposition 5.2. Let $\bar{x}_{i}$ be the greatest point such that $g_{i}\left(\bar{x}_{i}\right)=0$. The value functions $V_{i}, i=1,2$, and the corresponding optimal thresholds satisfy the limiting properties

$$
\lim _{\lambda \rightarrow \infty} V_{i}(x)=V^{\infty}(x):= \begin{cases}g_{1}(x) & \text { if } x \geq \bar{x}_{1} \\ 0 & \text { if } x \in\left(\bar{x}_{2}, \bar{x}_{1}\right) \\ g_{2}(x) & \text { if } x \leq \bar{x}_{2} .\end{cases}
$$

and

$$
\lim _{\lambda \rightarrow 0} V_{i}(x)=V(x) \quad \text { and }\left\{\begin{array}{l}
\lim _{\lambda \rightarrow 0} z_{i}^{*}=z^{*} \\
\lim _{\lambda \rightarrow 0} y_{i}^{*}=y^{*} .
\end{array}\right.
$$

Proof. We will prove the proposition only for Game 1; Game 2 is handled similarly. Let us first prove the case $\lambda \rightarrow \infty$. Recall from (2.4) and (2.5) that the value of the Game 1 reads as

$$
V_{1}(x)=\sup _{\tau \in \mathcal{T}_{1}} \inf _{\gamma \in \mathcal{T}_{0}} \Pi_{1}(x, \tau, \gamma)=\inf _{\gamma \in \mathcal{T}_{0}} \sup _{\tau \in \mathcal{T}_{1}} \Pi_{1}(x, \tau, \gamma)
$$

where $\Pi_{1}(x, \tau, \gamma)=\mathbf{E}_{x}\left\{e^{-r(\tau \wedge \gamma)}\left[g_{1}\left(X_{\tau}\right) \mathbf{1}_{\{\tau<\gamma\}}+g_{2}\left(X_{\gamma}\right) \mathbf{1}_{\{\tau>\gamma\}}+g_{3}\left(X_{\gamma}\right) \mathbf{1}_{\{\tau=\gamma\}}\right] \mathbf{1}_{\{\tau \wedge \gamma \leq T\}}\right\}$. Letting $\lambda \rightarrow$ $\infty$, we see that

$$
\begin{aligned}
& \Pi_{1}(x, \tau, \gamma)=0, \quad \text { if } \tau, \gamma>0 \\
& \Pi_{1}(x, \tau, \gamma)=g_{1}(x), \quad \text { if } \tau=0<\gamma \\
& \Pi_{1}(x, \tau, \gamma)=g_{2}(x), \quad \text { if } \tau>0=\gamma \\
& \Pi_{1}(x, \tau, \gamma)=g_{3}(x), \quad \text { if } \tau=0=\gamma
\end{aligned}
$$

In light of these findings, let us show that the claimed function $V^{\infty}$ is indeed the saddle point solution when $\lambda$ approaches to infinity. There are three cases to be considered depending whether $x \leq \bar{x}_{2}, x \in\left(\bar{x}_{2}, \bar{x}_{1}\right)$ or $x \geq \bar{x}_{1}$. (Note that since $g_{2} \geq g_{1}$, we always have $\bar{x}_{2} \leq \bar{x}_{1}$.) 
Let $x \leq \bar{x}_{2}$. Now $g_{1}(x) \leq g_{3}(x) \leq g_{2}(x) \leq 0$ and so we can check straightforwardly, using (5.4), that $\sup _{\tau \in \mathcal{T}_{1}} \inf _{\gamma \in \mathcal{T}_{0}} \Pi_{1}(x, \tau, \gamma)=g_{2}(x)=\inf _{\gamma \in \mathcal{T}_{0}} \sup _{\tau \in \mathcal{T}_{1}} \Pi_{1}(x, \tau, \gamma)$. The same reasoning applies also to the cases $x \in\left(\bar{x}_{2}, \bar{x}_{1}\right)$ and $x \geq \bar{x}_{1}$, and the claimed limiting property follows.

Next we turn our eyes on the case $\lambda \rightarrow 0$. Since $g_{1}^{+} \in L_{1}^{r}$, we find that $\lambda\left(R_{r+\lambda} g_{1}^{+}\right)(x) \rightarrow 0$ as $\lambda \rightarrow 0$ for all $x \in \mathbf{R}_{+}$. Given this limiting property together with the definition of $V_{1}$ in (3.11), we find that the claimed limiting property holds. Finally given the convergence result of value function $V_{1}$, the claimed convergence results hold also for the thresholds $z_{1}^{*}$ and $y_{1}^{*}$.

It is interesting to observe that the values of Game 1 and Game 2 are the same at the limit $\lambda \rightarrow 0$ and also at $\lambda \rightarrow \infty$. In the limit $\lambda \rightarrow 0$, this result is intuitively plausible: If the expected waiting time for the Poisson process to jump is infinite, the game will not expire unexpectedly, and as a result we get the solution of an infinite horizon game. Also the limit $\lambda \rightarrow \infty$ has a natural explanation: There is no advantage of observing the jump, since both players already know that the jump will occur at the time zero.

\section{Explicit Example with Geometric Brownian Motion}

We illustrate the main results of the study in this section with an explicit example. Let the underlying diffusion be geometric Brownian motion, that is, let $X$ be the solution of the Itô equation

$$
d X_{t}=\mu X_{t} d t+\sigma X_{t} d W_{t}
$$

where $W$ is the Wiener process. Furthermore we assume that $r>\mu$. Further let $g_{1}(x)=x-c_{1}$ and $g_{2}(x)=x-c_{2}$ and assume that $c_{1}>c_{2}>0$, so that $g_{2}>g_{1}$. Given this setup, we find that $\left(R_{r+\lambda} g_{i}\right)(x)=$ $\frac{x}{r+\lambda-\mu}-\frac{c_{i}}{r+\lambda}$.

In this case the decreasing and increasing fundamental solutions of the ordinary second order differential equation $(\mathcal{A}-\beta) u=0$ are $\varphi_{\beta}(x)=x^{\gamma_{1}^{\beta}}$ and $\psi_{\beta}(x)=x^{\gamma_{2}^{\beta}}$ respectively. Here

$$
\gamma_{i}^{\beta}=\frac{1}{\sigma^{2}}\left(\frac{1}{2} \sigma^{2}-\mu+(-1)^{i} \sqrt{\left(\frac{1}{2} \sigma^{2}-\mu\right)^{2}+2 \sigma^{2} \beta}\right),
$$

for $i=1,2$, are the solutions of the characteristic equation $\frac{1}{2} \sigma^{2} \gamma_{i}\left(\gamma_{i}-1\right)+\mu \gamma_{i}-r=0$. Finally, the scale density reads as $S^{\prime}(x)=x^{-\frac{2 \mu}{\sigma^{2}}}$. 
6.1. Game 1 has a solution. We know that $\left(R_{r+\lambda} g_{1}^{+}\right)(x)$ satisfies the differential equation $\frac{1}{2} \sigma^{2} x^{2}\left(R_{r+\lambda} g_{1}^{+}\right)^{\prime \prime}+$ $\mu x\left(R_{r+\lambda} g_{1}^{+}\right)^{\prime}-(r+\lambda)\left(R_{r+\lambda} g_{1}^{+}\right)=-g_{1}^{+}$. Therefore $\left(R_{r+\lambda} g_{1}^{+}\right)$satisfies the following conditions:

$$
\left(R_{r+\lambda} g_{1}^{+}\right)= \begin{cases}a_{1} \psi_{r+\lambda}(x)+a_{2} \varphi_{r+\lambda}(x) & \text { if } x \leq c_{1} \\ a_{3} \psi_{r+\lambda}(x)+a_{4} \varphi_{r+\lambda}(x)+\left(R_{r+\lambda} g_{1}\right)(x) & \text { if } x>c_{1} .\end{cases}
$$

Since $\left(R_{r+\lambda} g_{1}^{+}\right)(0+) \neq \infty$, we must have $a_{2}=0$ and since $\lim _{x \rightarrow \infty}\left(\left(R_{r+\lambda} g_{1}^{+}\right)(x)-\left(R_{r+\lambda} g_{1}\right)(x)\right)=0+$, we must have $a_{3}=0$. Furthermore $\left(R_{r+\lambda} g_{1}^{+}\right)$is continuous and differentiable. Thus the coefficients $a_{1}$ and $a_{4}$ can be solved from conditions $\lim _{x \rightarrow c_{1}+}\left(R_{r+\lambda} g_{1}^{+}\right)(x)=\lim _{x \rightarrow c_{1}-}\left(R_{r+\lambda} g_{1}^{+}\right)(x)$ and $\lim _{x \rightarrow c_{1}+}\left(R_{r+\lambda} g_{1}^{+}\right)^{\prime}(x)=$ $\lim _{x \rightarrow c_{1}-}\left(R_{r+\lambda} g_{1}^{+}\right)^{\prime}(x)$. It is a matter of elementary calculation to show that

$$
\begin{aligned}
& a_{1}=\frac{1}{\psi_{r+\lambda}\left(c_{1}\right)}\left(\left(R_{r+\lambda} g_{1}\right)\left(c_{1}\right)+\frac{\left(R_{r+\lambda} g_{1}\right)^{\prime}\left(c_{1}\right) \psi_{r+\lambda}\left(c_{1}\right)-\left(R_{r+\lambda} g_{1}\right)\left(c_{1}\right) \psi_{r+\lambda}^{\prime}\left(c_{1}\right)}{\varphi_{r+\lambda}\left(c_{1}\right) \psi_{r+\lambda}^{\prime}\left(c_{1}\right)-\varphi_{r+\lambda}^{\prime}\left(c_{1}\right) \psi_{r+\lambda}\left(c_{1}\right)} \varphi_{r+\lambda}\left(c_{1}\right)\right) \\
& a_{4}=\frac{\left(R_{r+\lambda} g_{1}\right)^{\prime}\left(c_{1}\right) \psi_{r+\lambda}\left(c_{1}\right)-\left(R_{r+\lambda} g_{1}\right)\left(c_{1}\right) \psi_{r+\lambda}^{\prime}\left(c_{1}\right)}{\varphi_{r+\lambda}\left(c_{1}\right) \psi_{r+\lambda}^{\prime}\left(c_{1}\right)-\varphi_{r+\lambda}^{\prime}\left(c_{1}\right) \psi_{r+\lambda}\left(c_{1}\right)} .
\end{aligned}
$$

Next we show that the presented setup satisfies the sufficient conditions of Theorem 3.7. Since $\mathcal{G}_{r} g_{i}(x)=$ $(\mu-r) x+r c_{i}$, for $i=1,2$, we find that $\mathcal{G}_{r} g_{i} \in \mathcal{L}_{1}^{r}$, for $i=1,2$. The assumption $c_{1}>c_{2}$ implies that $\mathcal{G}_{r} g_{1}>\mathcal{G}_{r} g_{2}$ - thus the conditions (1) and (3) in Theorem (3.7) hold. Moreover, since we assumed $r>\mu$, we have that $\gamma_{2}^{r}>1$, therefore $\frac{g_{i}(x)}{\psi_{r}(x)}=x^{1-\gamma_{2}^{r}}+c_{i} x^{-\gamma_{2}^{r}}$, for $i=1,2$, satisfy condition (2) in Theorem 3.7. Finally for the condition (3.13) recall that $\hat{g}_{i}=g_{i}-\lambda\left(R_{r+\lambda} g_{1}^{+}\right)$. Thus $\mathcal{G}_{r+\lambda} \hat{g}_{i}=\mathcal{G}_{r} g_{i}+\lambda\left(g_{1}^{+}-g_{i}\right)$ and we get

$$
\begin{aligned}
& \mathcal{G}_{r+\lambda} \hat{g}_{1}(x)= \begin{cases}(\mu-r-\lambda) x+(r+\lambda) c_{1} & \text { if } x<c_{1}, \\
(\mu-r) x+r c_{1} & \text { if } x \geq c_{1},\end{cases} \\
& \mathcal{G}_{r+\lambda} \hat{g}_{2}(x)= \begin{cases}(\mu-r-\lambda) x+(r+\lambda) c_{2} & \text { if } x<c_{1}, \\
(\mu-r) x+(r+\lambda) c_{2}-\lambda c_{1} & \text { if } x \geq c_{1} .\end{cases}
\end{aligned}
$$

From these expressions we see that the condition (3.13) holds and $\hat{x}_{1}>c_{1}$.

It follows that we can apply Theorem 3.7 and, consequently, that there exists a unique pair $\left(z_{1}^{*}, y_{1}^{*}\right)$ satisfying the necessary optimality conditions (3.12). If $z_{1}^{*}<c_{1}$, the conditions (3.12) can be written as (to simplify notation, we write $\gamma_{i}:=\gamma_{i}^{r+\lambda}$ )

$$
\left\{\begin{aligned}
\left(y^{\gamma_{1}} \lambda a_{4}\left(\gamma_{1}-\gamma_{2}\right)+\frac{y\left(\gamma_{2}-1\right)(r-\mu)}{r+\lambda-\mu}-\frac{r \gamma_{2} c_{1}}{r+\lambda}\right) y^{\gamma_{2}+\frac{2 \mu}{\sigma^{2}}-1} & =z^{\gamma_{2}+\frac{2 \mu}{\sigma^{2}}-1}\left(z\left(\gamma_{2}-1\right)-\gamma_{2} c_{2}\right) \\
\left(\frac{y\left(\gamma_{1}-1\right)(r-\mu)}{r+\lambda-\mu}-\frac{r \gamma_{1} c_{1}}{r+\lambda}\right) y^{\gamma_{1}+\frac{2 \mu}{\sigma^{2}}-1} & =z^{\gamma_{1}+\frac{2 \mu}{\sigma^{2}}-1}\left(z\left(\gamma_{1}-1\right)-\gamma_{1} c_{2}+z^{\gamma_{2}} \lambda a_{1}\left(\gamma_{2}-\gamma_{1}\right)\right) .
\end{aligned}\right.
$$


If, on the other hand, $z_{1}^{*} \geq c_{1}$, conditions (3.12) take the form

$$
\left\{\begin{array}{c}
\left(y^{\gamma_{1}} \lambda a_{4}\left(\gamma_{1}-\gamma_{2}\right)+\frac{y\left(\gamma_{2}-1\right)(r-\mu)}{r+\lambda-\mu}-\frac{r \gamma_{2} c_{1}}{r+\lambda}\right) y^{\gamma_{2}+\frac{2 \mu}{\sigma^{2}}-1}=z^{\gamma_{2}+\frac{\mu}{\sigma^{2}}-1}\left(\frac{\left(\gamma_{2}-1\right)(r-\mu) z}{r+\lambda-\mu}+\frac{\gamma_{2} \lambda c_{1}}{r+\lambda}-\gamma_{2} c_{2}+z^{\gamma_{1}}\left(\gamma_{1}-\gamma_{2}\right) \lambda a_{4}\right) \\
\left(\frac{y\left(\gamma_{1}-1\right)(r-\mu)}{r+\lambda-\mu}-\frac{r \gamma_{1} c_{1}}{r+\lambda}\right) y^{\gamma_{1}+\frac{2 \mu}{\sigma^{2}}-1}=z^{\gamma_{1}+\frac{2 \mu}{\sigma^{2}}-1}\left(\frac{z\left(\gamma_{1}-1\right)(r-\mu)}{r+\lambda-\mu}+\frac{\gamma_{1} \lambda c_{1}}{r+\lambda}-\gamma_{1} c_{2}\right)
\end{array}\right.
$$

Indeed, the function $\left(R_{r+\lambda} g_{1}^{+}\right)$is piecewise linear with conversion point at $c_{1}$. Furthermore $y_{1}^{*}>\hat{x}_{1}>c_{1}$ (see Proposition 3.3), but we do not know whether $z_{1}^{*}<c_{1}$ or the other way around. Therefore we have two alternative formulation for (3.12). Nevertheless, only one of these have solution, since Theorem 3.7 guarantees the uniqueness of the solution. Furthermore at the point $z=c_{1}$, these two pair of equations become the same. Unfortunately solving the optimal boundaries from these equations explicitly does not seem to be possible. Therefore we illustrate the results numerically. But before that, let us see through the solvability of Game 2 .

6.2. Game 2 has a solution. Similarly to Game 1, we find that

$$
\left(R_{r+\lambda} g_{2}^{-}\right)(x)= \begin{cases}a_{5} \psi_{r+\lambda}(x)+\left(R_{r+\lambda} g_{2}\right)(x) & \text { if } x<c_{2} \\ a_{6} \varphi_{r+\lambda}(x) & \text { if } x \geq c_{2},\end{cases}
$$

where

$$
\begin{aligned}
& a_{5}=\frac{1}{\psi_{r+\lambda}\left(c_{2}\right)}\left(-\left(R_{r+\lambda} g_{2}\right)\left(c_{2}\right)+\frac{\left(R_{r+\lambda} g_{2}\right)^{\prime}\left(c_{2}\right) \psi_{r+\lambda}\left(c_{2}\right)-\left(R_{r+\lambda} g_{2}\right)\left(c_{2}\right) \psi_{r+\lambda}^{\prime}\left(c_{2}\right)}{\varphi_{r+\lambda}^{\prime}\left(c_{2}\right) \psi_{r+\lambda}\left(c_{2}\right)-\varphi_{r+\lambda}\left(c_{2}\right) \psi_{r+\lambda}^{\prime}\left(c_{2}\right)} \varphi_{r+\lambda}\left(c_{2}\right)\right) \\
& a_{6}=\frac{\left(R_{r+\lambda} g_{2}\right)^{\prime}\left(c_{2}\right) \psi_{r+\lambda}-\left(R_{r+\lambda} g_{2}\right)\left(c_{2}\right) \psi_{r+\lambda}^{\prime}\left(c_{2}\right)}{\varphi_{r+\lambda}^{\prime}\left(c_{2}\right) \psi_{r+\lambda}\left(c_{2}\right)-\varphi_{r+\lambda}\left(c_{2}\right) \psi_{r+\lambda}^{\prime}\left(c_{2}\right)} .
\end{aligned}
$$

In particular $a_{5}, a_{6}<0$.

Next, we verify that the sufficient conditions in Theorem 4.5 hold. We already showed with the Game 1 that the conditions (1)-(3) hold, so it suffices to check whether the condition (4.5) holds. Recall that $\check{g}_{i}=g_{i}-\lambda\left(R_{r+\lambda} g_{2}^{-}\right)$so that $\mathcal{G}_{r+\lambda} \check{g}_{1}=\mathcal{G}_{r} g_{i}+\lambda\left(g_{2}^{-}-g_{i}\right)$. Thus

$$
\begin{gathered}
\mathcal{G}_{r+\lambda} \check{g}_{1}(x)= \begin{cases}(\mu-r) x+(r+\lambda) c_{1}-\lambda c_{2} & \text { if } x<c_{2}, \\
(\mu-r-\lambda) x+(r+\lambda) c_{1} & \text { if } x \geq c_{2},\end{cases} \\
\mathcal{G}_{r+\lambda} \check{g}_{2}(x)= \begin{cases}(\mu-r) x+r c_{2} & \text { if } x<c_{2}, \\
(\mu-r-\lambda) x+(r+\lambda) c_{2} & \text { if } x \geq c_{2} .\end{cases}
\end{gathered}
$$

From these expressions we see that the condition (4.5) holds and $\check{x}_{1}>c_{2}$. 
Again, we can apply Theorem 4.5 and there exists a unique pair $\left(z_{2}^{*}, y_{2}^{*}\right)$ which satisfies the necessary optimality condition (4.4). This time, if $z_{2}^{*}<c_{2}$, the condition can be written as (to simplify notation we write $\left.\gamma_{i}:=\gamma_{i}^{r+\lambda}\right)$

$$
\left\{\begin{aligned}
\left(y^{\gamma_{1}}\left(\gamma_{1}-\gamma_{2}\right) \lambda a_{6}+y\left(\gamma_{2}-1\right)-\gamma_{2} c_{1}\right) y^{\gamma_{2}+\frac{2 \mu}{\sigma^{2}}-1} & =z^{\gamma_{2}+\frac{2 \mu}{\sigma^{2}}-1}\left(\frac{z(r-\mu)\left(1-\gamma_{2}\right)}{r+\lambda-\mu}-\frac{r \gamma_{2} c_{2}}{r+\lambda}\right) \\
\left(y\left(\gamma_{1}-1\right)-\gamma_{1} c_{1}\right) y^{\gamma_{1}+\frac{2 \mu}{\sigma^{2}}-1} & =z^{\gamma_{1}+\frac{2 \mu}{\sigma^{2}}-1}\left(\frac{z(r-\mu)\left(\gamma_{1}-1\right)}{r+\lambda-\mu}-\frac{r \gamma_{1} c_{2}}{r+\lambda}+z^{\gamma_{2}}\left(\gamma_{2}-\gamma_{1}\right) \lambda a_{5}\right) .
\end{aligned}\right.
$$

If, on the other hand, $z_{2}^{*} \geq c_{2}$, the condition (4.4) takes the form

$$
\left\{\begin{array}{c}
\left(y^{\gamma_{1}}\left(\gamma_{1}-\gamma_{2}\right) \lambda a_{6}+y\left(\gamma_{2}-1\right)-\gamma_{2} c_{1}\right) y^{\gamma_{2}+\frac{2 \mu}{\sigma^{2}}-1}=z^{\gamma_{2}+\frac{2 \mu}{\sigma^{2}}-1}\left(z\left(\gamma_{2}-1\right)-\gamma_{2} c_{2}+z^{\gamma_{1}}\left(\gamma_{1}-\gamma_{2}\right) \lambda a_{6}\right) \\
\left(y\left(\gamma_{1}-1\right)-\gamma_{1} c_{1}\right) y^{\gamma_{1}+\frac{2 \mu}{\sigma^{2}}-1}=z^{\gamma_{1}+\frac{2 \mu}{\sigma^{2}}-1}\left(z\left(\gamma_{1}-1\right)-\gamma_{1} c_{2}\right) .
\end{array}\right.
$$

Similarly to Game 1, we know that $y_{2}^{*}>\check{x}_{1}>c_{2}$ (cf. Proposition 4.2), but we do not know whether $z_{2}^{*}<c_{2}$ or not. Therefore we have two alternative formulation of (4.4), but only one of these have a solution. Again, solving the optimal boundaries from these equations explicitly does not seem to be possible and so we are prompted to do numerical illustrations.

6.3. Numerical illustration. To illustrate the optimal characteristics numerically, we fix the parameter configuration $\mu=0.03, r=0.08, \sigma=0.35, c_{1}=3, c_{2}=2$ and $\lambda=0.1$. Under this choice, the value functions for Game 1 and Game 2 are given in Figure 1(a)-(b).

(a)

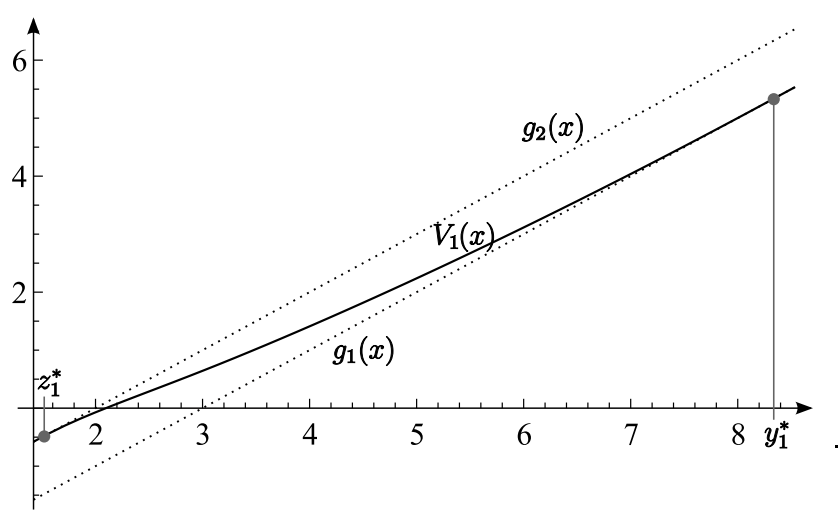

(b)

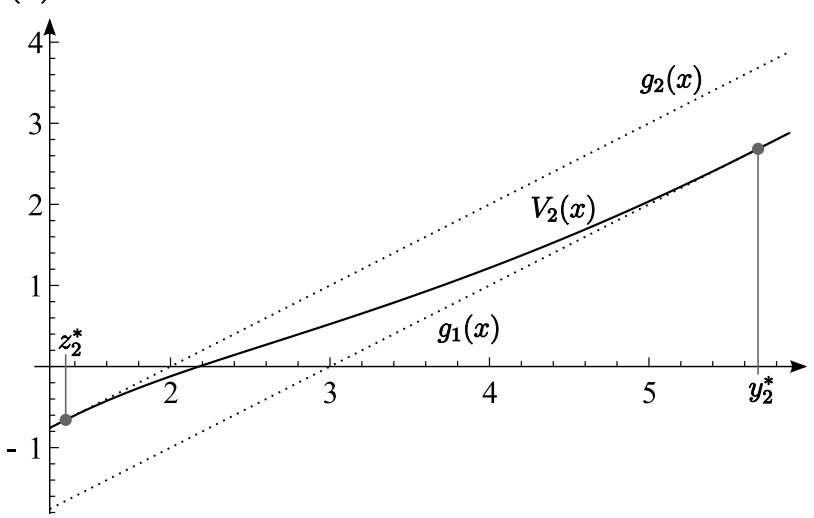

Figure 1. (a) The solution of Game 1; (b) The solution of Game 2. Now $\left(z_{1}^{*}, y_{1}^{*}\right)=(1.52,8.34)$, whereas $\left(z_{2}^{*}, y_{2}^{*}\right)=(1.34,5.68)$. For comparison in infinite horizon game $\left(z^{*}, y^{*}\right)=(1.60,8.99)$ so that now $z_{2}^{*}<z_{1}^{*}<z^{*}$ and $y_{2}^{*}<y_{1}^{*}<y^{*}$.

The values $V, V_{1}, V_{2}$ and $V_{3}$ are compared graphically in Figure 2, recall the definition of $V$ and $V_{3}$ from Section 5 . 


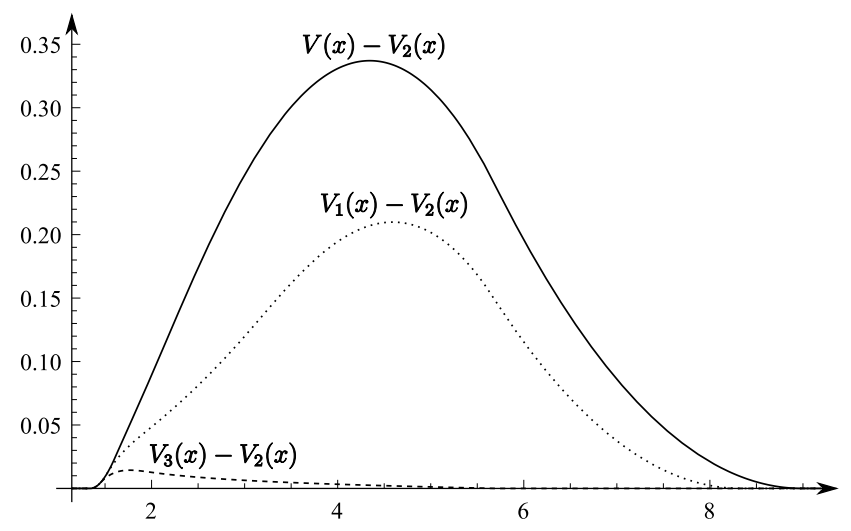

Figure 2. The differences $V-V_{2}, V_{1}-V_{2}$ and $V_{3}-V_{2}$.

In line with Proposition 5.1, we observe that the inequalities $V \geq V_{1} \geq V_{3} \geq V_{2}$ hold in this case. We point out that $V \geq V_{1}$ in this case even though $g_{2}$ takes also negative values. The values $V, V_{1}$ and $V_{2}$ appear to differ quite significantly from each others, which indicates that the mere existence of the expiry time and the inside information on it can have substantial impact on the optimal exercise rule. For example, if $x=4$ for the given parameters, we have $V(4) \approx 1.55$ and $V_{1}(4) \approx 1.41$ the difference being 0.14 , so that $V(4)$ is about $10 \%$ greater. However, we observe that the value $V_{3}$ does not differ much from $V_{2}$. This means that in this example when the issuer have inside knowledge about Poisson clock (Game 2), she rarely takes advantage of this information. This, in turn, is because she exercises at the jump time $T$ only if $g_{2}\left(X_{T}\right)<0$. This happens rarely, since $g_{2}$ is usually positive.

In Figure 3 we illustrate the sensitivities of the exercise thresholds with respect to parameters $\sigma$ and $\lambda$ in Game 1 and in the infinite horizon game. We notice that the order of the lower thresholds change as $\sigma$ increases. This is possible, since $g_{2}$ takes also negative values (cf. Proposition 5.1). Moreover we see that as $\sigma$ increases, the continuation region gets wider. This result is in line with the literature. Furthermore, we observe that the continuation region shrinks as $\lambda$ increases which is again natural in the current example. In particular, the issuer lets her exercise threshold grow towards $c_{2}$ so that she could increase her chances of exercising with negative payoff.

6.4. Counterexample for inequalities $z_{1}^{*} \leq z^{*}$ and $y_{1}^{*} \leq y^{*}$. In Proposition 5.1 we prove that if $g_{2} \geq 0$, then for the optimal stopping boundaries we have the inequalities $z_{1}^{*} \leq z^{*}$ and $y_{1}^{*} \leq y^{*}$ and for the values we 

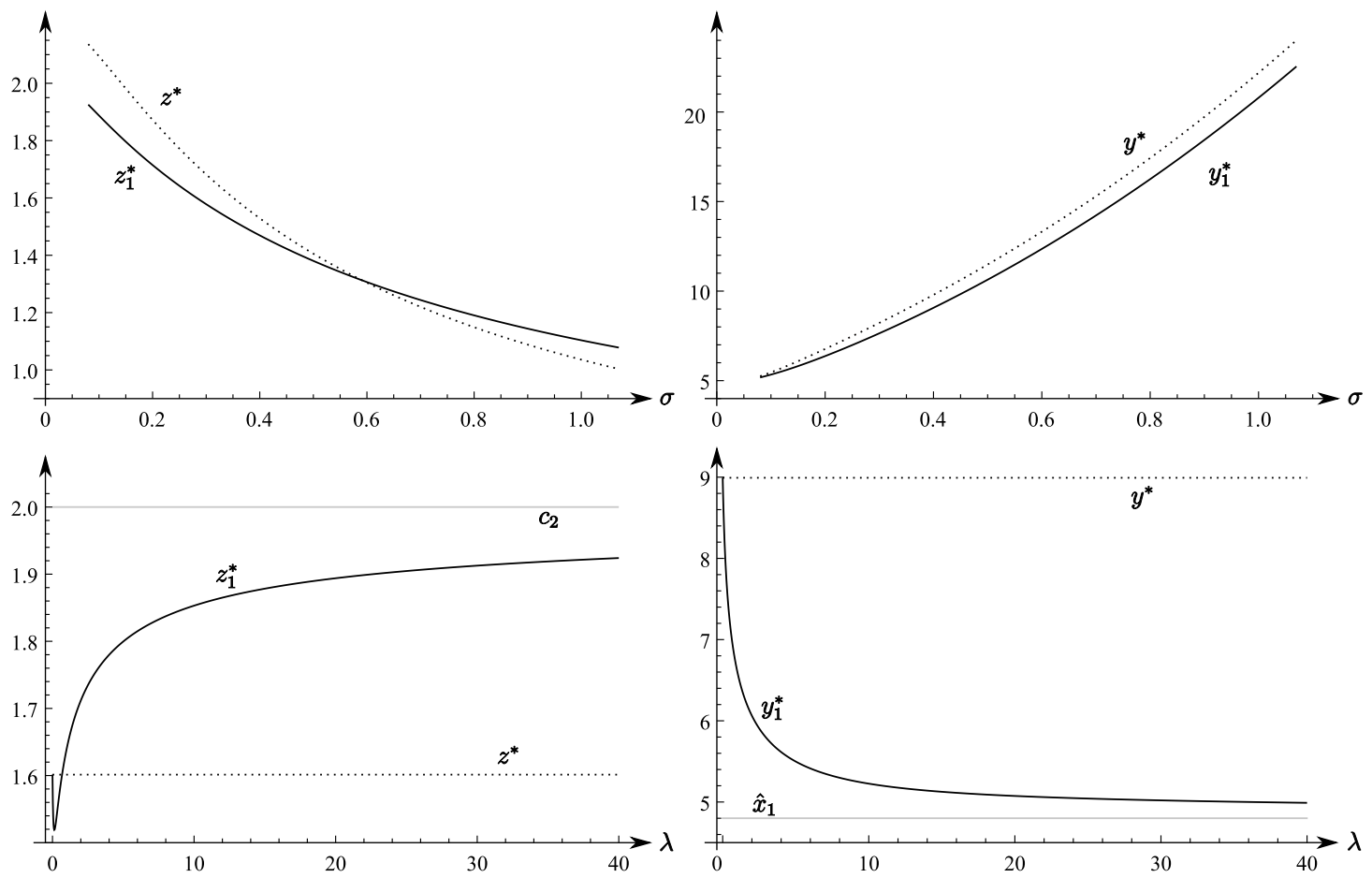

FiguRE 3. The changes of thresholds in Game 1 and in infinite horizon game, when changing $\sigma$ and $\lambda$.

have $V(x) \geq V_{1}(x)$. In this subsection we show that if $g_{2}$ is allowed to be negative, then these inequalities are not necessary true, a hint of this can also be seen from Figure 3.

Let the underlying diffusion still be a geometric Brownian motion and the parameter configuration as $\mu=0.03, \sigma=1.0, \lambda=0.1$ and $r=0.08$. Furthermore, let $g_{1}=\sqrt[3]{x}-3$ and $g_{2}=\sqrt{x}-2$; in particular, $g_{2}>g_{1}$. It is a straightforward task to check that there exist unique saddle point solutions for Game 1, Game 2 and infinite time horizon game and that the optimal thresholds read as $\left(z_{1}^{*}, y_{1}^{*}\right) \approx(0.56,44.7)$; $\left(z_{2}^{*}, y_{2}^{*}\right) \approx(0.24,39.4)$ and $\left(z^{*}, y^{*}\right) \approx(0.21,30.0)$. Now contrary to Proposition 5.1(B), we have $z_{1}^{*}>z^{*}$ and $y_{1}^{*}>y^{*}$. Moreover, we have also that $z_{2}^{*}>z^{*}$ and $y_{2}^{*}>y^{*}$. On the other hand, the boundaries of Game 2 are lower than the ones of Game 1, see Proposition 5.1(A). Moreover, we find that $V(x) \leq V_{2}(x) \leq V_{1}(x)$ which is illustrated in Figure 4.

It is interesting to observe that the value of a random time horizon game can dominate the value of an infinite horizon game. In fact, it can be that the infinite horizon game can have the smallest value of the games considered in this paper, which seems first rather counterintuitive. However, this is all due to the "sufficient negativeness" of $g_{2}$. Indeed, as the game will end almost surely in finite time, the issuers chances of exercising with a very negative payoff is reduced in comparison to the infinite horizon game. 


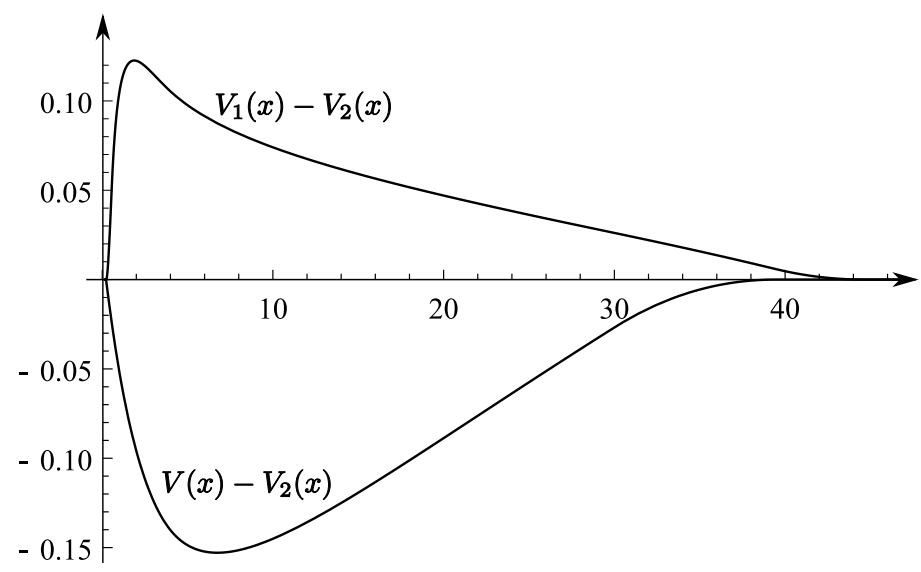

Figure 4. The differences $V-V_{2}$ and $V_{1}-V_{2}$. We have $V \leq V_{2} \leq V_{1}$ in contrast to Proposition 5.1.

Acknowledgements: The authors thank Prof. Fred Espen Benth for helpful discussions.

\section{REFERENCES}

[1] Alvarez, L. H. R. A Class of Solvable Stopping Games, 2008, Appl. Math. Optim., 58, 291 - 314

[2] Alvarez, L. H. R. Minimum Guaranteed Payments and Costly Cancellation Rights: A Stopping Game Perspective, 2010, Math. Finance, 20/4, $733-751$

[3] Borodin, A. and Salminen P. Handbook on Brownian Motion - Facts and Formula, 2002, Birkhäuser, Basel

[4] Carr, P. Randomization and the American Put, 1998, Rev. Finan. Stud., 11/3, 597 - 626

[5] Dynkin, E. Markov Processes II, 1965, Springer-Verlag, Berlin

[6] Dynkin, E. Game Variant of a problem of Optimal Stopping, 1969, Soviet Math. Dokl., 10, 270 - 274

[7] Ekström, E. Properties of game options, 2006, Math. Methods Oper. Res., 63/2, 221 - 238

[8] Ekström, E. and Villeneuve, S. On The Value of Optimal Stopping Games, 2006, Ann. Appl. Probab., 16, 1576 - 1596

[9] Ekström, E. and Peskir, G. Optimal Stopping Games for Markov Processes, 2008, SIAM J. Control Optim., 47/2, 684 702

[10] Jansons, K. M. and Rogers, L. C. G. Probability theory and polymer physics, 1991, J. Statist. Phys., 65, 139 - 165

[11] Johnson, T. C. and Zervos, M. The solution to a second order linear ordinary differential equation with a non-homogeneous term that is a measure, 2007, Stochastics, 79, $363-382$

[12] Kifer, Y. Game Options, 2000, Finance Stochastics, 4, $443-463$

[13] Kyprianou, A. E. Some calculations for Israeli options, 2004, Finance Stochastics, 8, 73 - 86

[14] Kyprianou, A. E. and Pistorius, M. R. Perpetual options and Canadization through fluctuation theory, 2003, Ann. Appl. Probab., 13/3, $1077-1098$

[15] Lempa, J. A Note on Optimal Stopping of Diffusions with a Two-sided Optimal Rule, 2010, Oper. Res. Lett., $38,11-16$

[16] Øksendal, B. Stochastic Differential Equations, 5th Edition, 2000, Springer

[17] Peskir, G. Optimal Stopping Games and Nash Equilibrium, 2008, Theory Probab. Appl., 53/3, 623 - 638

[18] Protter, P. E. Stochastic Integration and Differential Equations, 2005, 2nd edition, Version 2.1, Springer-Verlag

[19] Salminen, P. Optimal Stopping of One-Dimensional Diffusions, 1985, Math. Nachr., 124, 85 - 101 